ESAIM: M2AN 47 (2013) 743-769

DOI: $10.1051 / \mathrm{m} 2 \mathrm{an} / 2012047$
ESAIM: Mathematical Modelling and Numerical Analysis

www.esaim-m2an.org

\title{
A DIFFUSE INTERFACE FRACTIONAL TIME-STEPPING TECHNIQUE FOR INCOMPRESSIBLE TWO-PHASE FLOWS WITH MOVING CONTACT LINES *
}

\author{
ABner J. SAlgado ${ }^{1}$
}

\begin{abstract}
For a two phase incompressible flow we consider a diffuse interface model aimed at addressing the movement of three-phase (fluid-fluid-solid) contact lines. The model consists of the Cahn Hilliard Navier Stokes system with a variant of the Navier slip boundary conditions. We show that this model possesses a natural energy law. For this system, a new numerical technique based on operator splitting and fractional time-stepping is proposed. The method is shown to be unconditionally stable. We present several numerical illustrations of this scheme.
\end{abstract}

Mathematics Subject Classification. 35Q30, 76D27, 76T99, 65N12.

Received February 17, 2012. Revised June 29, 2012

Published online March 29, 2013.

\section{INTRODUCTION}

The no-slip boundary condition is usually regarded as a cornerstone in fluid dynamics, and its applicability has been proved for diverse fluid flow problems. However, when dealing with two-phase flows it is of importance to accurately describe the displacement of the so-called three-phase contact line, that is, the points which are at the intersection of the solid boundary of the domain and the interface separating the two fluids. In this case, contrary to what is seen in experiments, the no-slip condition implies that the contact line does not move. This is known as the contact line problem (paradox) and it has recently been the subject of intense research and debate. The interested reader is referred to $[4,5,11,32,34,38,39]$ for an overview.

Let us elaborate on the ideas mentioned above. Consider the motion of an incompressible, viscous two-phase fluid, which can be described by the time-dependent Navier-Stokes equations,

$$
\left\{\begin{array}{l}
\rho_{t}+\nabla \cdot(\rho \mathbf{u})=0, \\
\rho\left(\mathbf{u}_{t}+\mathbf{u} \cdot \nabla \mathbf{u}\right)+\nabla \mathbf{p}-\nabla \cdot(\eta \mathbf{T}(\mathbf{u}))=\rho \mathbf{f}+\gamma H \mathbf{n}_{\Sigma} \delta_{\Sigma}, \\
\nabla \cdot \mathbf{u}=0
\end{array}\right.
$$

The unknowns are: $\rho>0$ - the density, the velocity field $u$ and the pressure $\mathrm{p}$; the parameter $\eta>0$ is the dynamic viscosity coefficient and $\mathbf{f}$ is an external driving force; $\Sigma$ is the interface between the two phases (which

\footnotetext{
Keywords and phrases. Navier Stokes, Cahn Hilliard, multiphase flow, contact line, fractional time-stepping.

* This material is based on work supported by NSF grants CBET-0754983 and DMS-0807811 and an AMS-Simons grant.

1 Department of Mathematics, University of Maryland, College Park, MD 20742, USA. abnersg@math.umd.edu
} 
is also a priori unknown, thus making this a free boundary problem), $\mathbf{n}_{\Sigma}$ its normal and $H$ its mean curvature; $\gamma$ is the surface tension coefficient. For a vector $\mathbf{v}$, we have denoted by $\mathbf{T}(\mathbf{v})$ the symmetric gradient $\nabla \mathbf{v}+(\nabla \mathbf{v})^{\top}$. The fluid occupies a bounded domain $\Omega$ in $\mathbb{R}^{d}$ with $d=2$ or 3 , and a solution to the above problem is sought over a bounded time interval $[0, T]$. We supplement this system with the initial conditions

$$
\left.\rho\right|_{t=0}=\left.\rho_{0}(x) \quad \mathrm{u}\right|_{t=0}=\mathrm{u}_{0}(x) .
$$

If we assume that the fluid is confined within solid walls, then we must impose the so-called impermeability condition

$$
\left.\mathbf{u} \cdot \mathbf{n}\right|_{\Gamma}=0
$$

where $\Gamma=\partial \Omega$ and $\mathbf{n}$ is the outer unit normal to $\Gamma$. This boundary condition implies that we do not need boundary conditions on the density or pressure. However, we must still prescribe a boundary condition on the tangential component of the velocity. Usually, the no-slip condition is assumed

$$
\left.\mathbf{u} \cdot \boldsymbol{\tau}\right|_{\Gamma}=\mathbf{U}(x, t) \cdot \tau
$$

where $\boldsymbol{\tau}$ is any vector tangent to $\Gamma$ and $\mathbf{U}$ is the wall velocity. Let us now consider the case of a non-moving container $(\mathbf{U} \equiv 0)$ and assume that at some instant part of the interface $\Sigma$ reaches the walls $\Gamma$. If that is the case, the no-slip boundary condition (1.4) implies then that $\Sigma \cap \Gamma$ (the contact line) will not move anymore. This is in contrast to what experience dictates.

The seminal work by Huh and Scriven [21] is one of the first references to approach systematically the contact line problem. They showed that the no-slip boundary condition implies that the shear stress and pressure have a non-integrable singularity at the contact line which, in turn, implies that the work done by the wall stresses and the viscous dissipation are infinite. They conclude that the no-slip condition is the cause of this conundrum and propose that some type of smoothing must be introduced to relax this condition. As a first variant they propose to consider the well-known Navier slip condition

$$
\beta(\mathbf{u}-\mathbf{U}) \cdot \boldsymbol{\tau}+\left.\eta(\mathbf{T}(\mathbf{u}) \cdot \mathbf{n}) \cdot \boldsymbol{\tau}\right|_{\Gamma}=0,
$$

where $\beta$ is the slip coefficient. In practice, this coefficient is very large, which explains why in many cases the no-slip condition is a good approximation. However, they realize that to obtain a consistent model one must also take into account the fact that there are additional interfacial stresses acting on the three-phase contact line $\Sigma \cap \Gamma$, see also [38].

Qian et al., first on the basis of molecular dynamics simulations ( $c f$. [31]), and then using variational principles (cf. [33]); have proposed the so-called generalized Navier boundary condition:

$$
\beta(\mathbf{u}-\mathbf{U}) \cdot \boldsymbol{\tau}+\eta(\mathbf{T}(\mathbf{u}) \cdot \mathbf{n}) \cdot \boldsymbol{\tau}+\left.\gamma\left(\cos \left(\theta_{d}\right)-\cos \left(\theta_{s}\right)\right) \mathbf{t} \cdot \boldsymbol{\tau} \delta_{\partial \Sigma}\right|_{\Gamma}=0,
$$

where $\delta_{\partial \Sigma}$ is a Dirac measure supported at the contact line, $\theta_{d}$ is the angle between $\Sigma$ and $\Gamma, \theta_{s}$ is the static contact angle, i.e. the angle $\Sigma$ and $\Gamma$ would make at equilibrium and is considered part of the data of the problem and $\mathbf{t}$ is the unit normal vector of $\Sigma$ restricted to $\Gamma$. Compared to the classical Navier slip boundary conditions, we have the extra term

$$
\gamma\left(\cos \left(\theta_{d}\right)-\cos \left(\theta_{s}\right)\right) \mathbf{t} \cdot \boldsymbol{\tau} \delta_{\partial \Sigma}
$$

which is the so-called uncompensated Young stress, and measures the difference between the dynamic contact angle and the static contact angle $\theta_{s}$.

In this work we consider a diffuse interface model of (1.1), (1.2), (1.3) and (1.6). We show that this system has an energy law and propose an efficient discretization scheme for it. Our model is a generalization of the one treated in [33], where only the case of a Stokes fluid is considered. In this respect, it is related to the models presented in [1] where, however, boundary conditions are not discussed. The energy law that we obtain for this model is similar to the ones obtained in the aforementioned references and, in addition, generalizes the one 
obtained in [14], Theorem 3.1, to the case of two-phase fluids with different densities. Moreover, this energy law is a variant of the one obtained in [15], Proposition 1, where the sharp interface version (1.6) of this boundary condition is considered. Concerning numerics, our approach is new in the sense that it extends the schemes of $[19,35]$ to the case of the generalized Navier boundary condition, and thus it proposes schemes that are more general than the ones considered in $[14,20]$, which are restricted to the matched density case.

The organization of this work is as follows: Section 2 introduces the diffuse interface model we shall be concerned with. In Section 3 we show that this system has an energy law. A time discrete scheme for our model is introduced in Section 4 and we show that this scheme is unconditionally stable. The space discretization is handled using Galerkin techniques in Section 5 and it is shown that the fully discrete scheme shares the same stability properties as the semi-discrete one. Finally, a series of numerical experiments aimed at illustrating the capabilities of the proposed algorithm are exposed in Section 6.

\section{A Diffuse interface MOdel For MOVing CONTACT Lines}

Let us present the model. To simplify notation, for any vector $\mathbf{v}$ defined on $\Gamma$, we denote

$$
\mathbf{v}_{\boldsymbol{\tau}}:=\mathbf{v}-(\mathbf{v} \cdot \mathbf{n}) \mathbf{n},
$$

similarly, for any smooth enough scalar field $\psi$ defined on $\Gamma$, we denote

$$
\partial_{\boldsymbol{\tau}} \psi:=(\nabla \psi)_{\boldsymbol{\tau}}
$$

On the basis of thermodynamical principles, [33] has obtained a diffuse interface variant of (1.6) for a flow governed by Stokes law. Repeating the steps provided in this reference, we can obtain a more general model which we now describe. The reader is referred to $[1,29]$ for details. Let us introduce the so-called phase field variable $\phi$, which serves as a marker, i.e. $\phi \approx \pm 1$ on each one of the phases, respectively. All material parameters that are phase dependent are then functions of $\phi$. For instance, we can define the density as

$$
\rho(\phi)=\frac{\rho_{1}-\rho_{2}}{2} \phi+\frac{\rho_{1}+\rho_{2}}{2} .
$$

The phase field variable $\phi$ satisfies

$$
\begin{cases}\phi_{t}+\mathrm{u} \cdot \nabla \phi=\nabla \cdot(M(\phi) \nabla \mu), & \text { in } \Omega, \\ M(\phi) \partial_{\mathbf{n}} \mu=0, & \text { on } \Gamma, \\ \alpha\left(\phi_{t}+\mathrm{u}_{\boldsymbol{\tau}} \partial_{\boldsymbol{\tau}} \phi\right)+L(\phi)=0, & \text { on } \Gamma .\end{cases}
$$

Here $M>0$ is a (phenomenological) mobility parameter and $\alpha$ is a relaxation parameter. The quantity $\mu$ is known as the chemical potential and is defined by

$$
\mu:=\gamma\left(\frac{1}{\delta} \mathcal{W}{ }^{\prime}(\phi)-\delta \Delta \phi\right)+\frac{1}{2} \rho^{\prime}(\phi)|\mathrm{u}|^{2},
$$

where the function $\mathcal{W}$ is the Ginzburg-Landau double well potential

$$
\mathcal{W}(r)=\frac{1}{4}\left(1-r^{2}\right)^{2}
$$

and $\delta$ is a parameter that characterizes the interface thickness. The boundary condition $M(\phi) \partial_{\mathbf{n}} \mu=0$ comes from impermeability. Finally,

$$
L(\phi):=\gamma\left(\delta \partial_{\mathbf{n}} \phi+\Theta_{f s}^{\prime}(\phi)\right),
$$

$\Theta_{f s}$ is the interfacial free energy density per unit area at the fluid-solid interface. A possible definition of the interfacial free energy is

$$
\Theta_{f s}(\phi)=\cos \left(\theta_{s}\right) \sin (\pi \phi / 2)+\frac{\gamma_{1,2}}{2 \gamma}
$$

where $\gamma_{1,2}$ is the difference of surface tensions between each one of the phases and the solid. 
Remark 2.1 (Static contact angle). It has been shown in [33] that minimization of the free energy with respect to $\phi$ leads to $L(\phi) \equiv 0$, from which we can recover Young's equation for the static contact angle, i.e.

$$
\gamma \Theta_{f s}\left(\phi_{+}\right)+\gamma \cos \theta_{s}=\gamma \Theta_{f s}\left(\phi_{-}\right)
$$

see [33], equation (4.6) for more details.

The velocity and pressure satisfy

$$
\begin{cases}\sigma(\phi)(\sigma(\phi) \mathbf{u})_{t}+(\rho(\phi) \mathbf{u} \cdot \nabla) \mathbf{u}+\frac{1}{2} \nabla \cdot(\rho(\phi) \mathbf{u}) \mathbf{u}-\nabla \cdot(\eta(\phi) \mathbf{T}(\mathbf{u}))+\nabla \mathbf{p}= & \\ \rho(\phi) \mathbf{f}+\mu \nabla \phi+\frac{1}{2} \rho^{\prime}(\phi) \phi_{t} \mathbf{u}, & \text { in } \Omega, \\ \nabla \cdot \mathbf{u}=0, & \text { in } \Omega, \\ \mathbf{u}_{\mathbf{n}}=0, \quad \beta(\phi)\left(\mathbf{u}_{\boldsymbol{\tau}}-\mathbf{U}\right)+\eta(\phi) \mathbf{T}(\mathbf{u})_{\mathbf{n} \boldsymbol{\tau}}=L(\phi) \partial_{\boldsymbol{\tau}} \phi, & \text { on } \Gamma .\end{cases}
$$

Here $\sigma(\phi):=\sqrt{\rho(\phi)}$. The surface tension is now expressed by the term $\mu \nabla \phi$, see $[22,35,37]$ for more details.

Remark 2.2 (Momentum equation). Notice that, as initially proposed in [18], we have modified the original form of the momentum equation

$$
\rho(\phi) \mathbf{u}_{t}+(\rho(\phi) \mathbf{u} \cdot \nabla) \mathbf{u}-\nabla \cdot(\eta(\phi) \mathbf{T}(\mathbf{u}))+\nabla \mathbf{p}=\rho(\phi) \mathbf{f}+\mu \nabla \phi,
$$

by adding to it

$$
\frac{1}{2}\left(\rho(\phi)_{t}+\nabla \cdot(\rho(\phi) \mathbf{u})\right) \mathbf{u}=0,
$$

i.e. a suitable multiple of the mass conservation equation. In this respect, we must mention that this step is also performed in [37], where the phase field Navier Stokes equations with no-slip boundary conditions are studied. The purpose of this reformulation is to take advantage of the following identity

$$
\int_{\Omega}[(\rho(\phi) \mathbf{v} \cdot \nabla) \mathbf{w}] \cdot \mathbf{w}+\frac{1}{2} \int_{\Omega} \nabla \cdot(\rho(\phi) \mathbf{v}) \mathbf{w} \cdot \mathbf{w}=0,
$$

for all $\left.\mathbf{v} \cdot \mathbf{n}\right|_{\Gamma}=0$. Notice that we do not require the fields to be solenoidal. This is important not just to obtain an energy law of this system but also in the design of efficient numerical schemes. In this respect, the reader is referred to [6], Section 2.3 where, for the standard Cahn Hilliard Navier Stokes problem, a numerical simulation is presented that illustrates the loss of stability that might occur if this modification is not accounted for.

Remark 2.3 (Diffuse interface model). Several remarks are in order about this model:

- Model (2.1)-(2.4) belongs to the general class of so-called phase field models, which have recently gained popularity in the literature. One of the main advantages of phase field formulations for the simulation of free surface flows is that, as opposed to interface tracking techniques (i.e. ALE formulations), topological changes can be handled automatically. In addition, derivation of such models is based on the physical principle of energy dissipation, which might not be the case with other interface capturing techniques as, say, level set methods.

- The derivation of the phase field version of the generalized Navier boundary condition introduced in (2.4) as well as its relation with the sharp interface counterpart presented in (1.6) is discussed in [32], Section 5.2.

- The model contains several parameters. The origin and values of $M, \alpha, \delta, \theta_{s}$ and $\beta$ are discussed in $[32,33]$.

\section{ENERGy LAW FOR THE GENERALIZED NAVIER BOUNDARY CONDITION}

The purpose of this section is to show that the Cahn Hilliard Navier Stokes equations with generalized Navier boundary conditions (2.1)-(2.4) satisfy an energy law. This is important not just from an analysis point of view, as it guarantees that, in a sense, the system is thermodynamically consistent (see [1]), but will also guide us in the construction of numerical schemes. To avoid irrelevant technicalities, let us assume that $\mathbf{f} \equiv 0$ and $\mathbf{U} \equiv 0$. 
Theorem 3.1 (Energy law). Let $\mathbf{f} \equiv 0$ and $\mathbf{U} \equiv 0$. Then the system (2.1)-(2.4) has the following energy law

$$
\begin{aligned}
\frac{\mathrm{d}}{\mathrm{d} t}\left\{\int _ { \Omega } \left[\frac{1}{2}|\sigma(\phi) \mathrm{u}|^{2}+\gamma\left(\frac{\delta}{2}|\nabla \phi|^{2}\right.\right.\right. & \left.\left.\left.+\frac{1}{\delta} \mathcal{W}(\phi)\right)\right]+\gamma \int_{\Gamma} \Theta_{f s}(\phi)\right\} \\
& +\int_{\Omega}\left(\eta(\phi)|\mathbf{T}(\mathrm{u})|^{2}+M(\phi)|\nabla \mu|^{2}\right)+\int_{\Gamma}\left(\beta(\phi)\left|\mathbf{u}_{\boldsymbol{\tau}}\right|^{2}+\alpha\left|\phi_{t}+\mathbf{u}_{\boldsymbol{\tau}} \partial_{\boldsymbol{\tau}} \phi\right|^{2}\right)=0 .
\end{aligned}
$$

Remark 3.2 (Physical interpretation of the energy law). The many terms in (3.1), can be given a physical interpretation. Consider first the term inside the time derivative:

$$
\int_{\Omega}\left[\frac{1}{2}|\sigma(\phi) \mathrm{u}|^{2}+\gamma\left(\frac{\delta}{2}|\nabla \phi|^{2}+\frac{1}{\delta} \mathcal{W}(\phi)\right)\right]+\gamma \int_{\Gamma} \Theta_{f s}(\phi) .
$$

The first term is the kinetic energy of the fluid; the second and third correspond to the interfacial energy; whereas the boundary integral term to the energy at the walls. The other terms can be characterized as dissipative mechanisms in the system. It is clear that $\int_{\Omega} \eta(\phi)|\mathbf{T}(\mathbf{u})|^{2}$ represents viscous dissipation; the term $\int_{\Omega} M(\phi)|\nabla \mu|^{2}$, as stated in [33], gives the composition diffusion in the bulk; and, finally, the boundary terms are associated with the loss mechanism related with the movement of the fluid at the walls. In other words, (3.1) expresses that the change in energy is due to dissipation.

Proof. (Theorem 3.1) Let us first multiply the momentum equation (first equation in (2.4)) by u and integrate over $\Omega$. Using (2.5) we obtain

$$
\frac{1}{2} \frac{\mathrm{d}}{\mathrm{d} t} \int_{\Omega}|\sigma(\phi) \mathrm{u}|^{2}-\int_{\Omega} \nabla \cdot(\eta(\phi) \mathbf{T}(\mathrm{u})) \cdot \mathrm{u}+\int_{\Omega} \nabla \mathrm{p} \cdot \mathbf{u}=\int_{\Omega} \mu \nabla \phi \cdot \mathbf{u}+\frac{1}{2} \int_{\Omega} \rho^{\prime}(\phi) \phi_{t}|\mathrm{u}|^{2} .
$$

The impermeability condition on the velocity and the incompressibility constraint give us

$$
\int_{\Omega} \nabla \mathrm{p} \cdot \mathrm{u}=\int_{\Gamma} \mathrm{pu}_{\mathbf{n}}-\int_{\Omega} \mathrm{p} \nabla \cdot \mathrm{u}=0
$$

Integration by parts and the impermeability condition give us

$$
\int_{\Omega} \nabla \cdot(\eta(\phi) \mathbf{T}(\mathrm{u})) \cdot \mathrm{u}=\int_{\Gamma} \eta(\phi) \mathbf{T}(\mathrm{u})_{\mathbf{n} \tau} \cdot \mathbf{u}_{\boldsymbol{\tau}}-\int_{\Omega} \eta(\phi)|\mathbf{T}(\mathrm{u})|^{2},
$$

so that the generalized Navier boundary condition gives us

$$
\begin{aligned}
\frac{1}{2} \frac{\mathrm{d}}{\mathrm{d} t} \int_{\Omega}|\sigma(\phi) \mathrm{u}|^{2}+\int_{\Omega} \eta(\phi)|\mathbf{T}(\mathrm{u})|^{2} & +\int_{\Gamma} \beta(\phi)\left|\mathbf{u}_{\boldsymbol{\tau}}\right|^{2} \\
& \quad+\alpha \int_{\Gamma}\left(\phi_{t}+\mathrm{u}_{\boldsymbol{\tau}} \partial_{\boldsymbol{\tau}} \phi\right) \mathrm{u}_{\boldsymbol{\tau}} \partial_{\boldsymbol{\tau}} \phi=\int_{\Omega} \mu \nabla \phi \cdot \mathrm{u}+\frac{1}{2} \int_{\Omega} \rho^{\prime}(\phi) \phi_{t}|\mathrm{u}|^{2} .
\end{aligned}
$$

Let us take now the first equation in (2.1), multiply it by $\mu$ and integrate it over $\Omega$. We obtain

$$
\int_{\Omega} \mu \phi_{t}+\int_{\Omega} \mu \nabla \phi \cdot \mathrm{u}=\int_{\Omega} \nabla \cdot(M(\phi) \nabla \mu) \mu .
$$

Integration by parts and the impermeability condition give us,

$$
\int_{\Omega} \nabla \cdot(M(\phi) \nabla \mu) \mu=-\int_{\Omega} M(\phi)|\nabla \mu|^{2} .
$$


Multiply the definition of the chemical potential $\mu$ by $-\phi_{t}$ and integrate over $\Omega$

$$
\begin{aligned}
-\int_{\Omega} \mu \phi_{t}=\gamma \delta \int_{\Omega} \Delta \phi \phi_{t}-\frac{\gamma}{\delta} \int_{\Omega} \mathcal{W}^{\prime}(\phi) \phi_{t}-\frac{1}{2} \int_{\Omega} \rho^{\prime}(\phi) \phi_{t}|\mathrm{u}|^{2} & = \\
& \gamma \delta \int_{\Gamma} \partial_{\mathbf{n}} \phi \phi_{t}-\frac{\gamma \delta}{2} \frac{\mathrm{d}}{\mathrm{d} t} \int_{\Omega}|\nabla \phi|^{2}-\frac{\gamma}{\delta} \frac{\mathrm{d}}{\mathrm{d} t} \int_{\Omega} \mathcal{W}(\phi)-\frac{1}{2} \int_{\Omega} \rho^{\prime}(\phi) \phi_{t}|\mathrm{u}|^{2},
\end{aligned}
$$

where to obtain the second equality we integrated by parts. The boundary condition on the phase field variable yields

$$
\gamma \delta \int_{\Gamma} \partial_{\mathbf{n}} \phi \phi_{t}=-\alpha \int_{\Gamma}\left(\phi_{t}+\mathrm{u}_{\boldsymbol{\tau}} \partial_{\boldsymbol{\tau}} \phi\right) \phi_{t}-\gamma \int_{\Gamma} \Theta_{f s}^{\prime}(\phi) \phi_{t}
$$

Adding the last four relations gives

$$
\begin{aligned}
\gamma \frac{\mathrm{d}}{\mathrm{d} t}\left[\int_{\Omega}\left(\frac{\delta}{2}|\nabla \phi|^{2}+\frac{1}{\delta} \mathcal{W}(\phi)\right)+\int_{\Gamma} \Theta_{f s}(\phi)\right]+\int_{\Omega} \mu \nabla \phi \cdot \mathrm{u}+ & \int_{\Omega} M(\phi)|\nabla \mu|^{2} \\
& +\alpha \int_{\Gamma}\left(\phi_{t}+\mathrm{u}_{\boldsymbol{\tau}} \partial_{\boldsymbol{\tau}} \phi\right) \phi_{t}
\end{aligned}
$$

Adding (3.2) and (3.3) we obtain the result.

Remark 3.3 (Energy law for matched density). A similar energy law has been obtained in [14]. This work, however, assumes that the density is constant which, clearly, simplifies the arguments. In particular, there is no need to introduce the modification of the momentum equation discussed in Remark 2.2.

\section{Time DiscretizATion AND StABILITy ANALYSis}

In this section, we study the time discretization of the phase field model introduced in Section 2. Our goal is to construct time discrete schemes that satisfy a discrete energy law which, in some appropriate sense, mimics (3.1). Moreover, these schemes should be computationally efficient.

Several works deal with the approximation of moving contact lines. See, for instance $[10,26,27,30,40,41]$. However, most of these works handle the contact line using the Navier slip boundary condition (1.5) or adding an empirical law that determines the tangential component of the velocity. A discussion on the validity and physical significance of these approaches (and of the generalized Navier boundary condition for that matter) is well beyond our scope of interest. We want to point out, however, that to the best of our knowledge, there are very few references where the generalized Navier boundary condition is used for the treatment of moving contact lines. [15] develops an ALE approach and [27] uses a volume of fluid formulation, coupled with interface tracking techniques. References $[14,20]$ present schemes similar to ours, but restricted to the matched density case.

The Cahn Hilliard Navier Stokes system (2.1)-(2.4) presents serious challenges for efficient numerical approximation. Even in the simpler case of standard boundary conditions, i.e. no-slip on the velocity and impermeability on the phase field, this system includes a fourth order evolution equation, coupled with the variable density Navier-Stokes equations. Without being exhaustive, we mention the following references: [13], [23-25] and [6,28]. The author of [13] proposes a discretization of the matched density case and shows that the solutions to his fully discrete scheme converge to an exact solution when the space and time discretization parameters tend to zero. The discretization scheme, however, is not the most amenable to computation, since it couples all the variables. References $[23,24]$ develop an operator splitting approach for the phase-field and chemical potential part, together with a coupled solver for the Navier-Stokes part. The work [25], under the assumption that the density does not depend on the phase, develops a nonlinear multigrid algorithm for the Cahn Hilliard system, which is coupled with a projection-type solver for the Navier-Stokes part. Finally, references $[6,28]$ are concerned 
with the discretization and analysis of a triphasic fluid. They propose unconditionally stable schemes and show convergence to exact solutions in the case of matched densities.

Amongst the most popular techniques used to solve the constant density Navier-Stokes equations are projection methods, which aim at decoupling the incompressibility constraint from the diffusion process. These methods are known to converge even in the case of open boundary conditions (see [17]). Moreover, efficient extensions of these schemes to the variable density or multiphase case have been recently proposed in $[19,35,37]$. On the basis of these observations, we apply a fractional time-stepping technique based on penalization of the divergence to the Navier Stokes part of the system. The Cahn Hilliard component is solved using an operator splitting approach, similar to [23-25].

To construct the time-discrete scheme, we replace the Ginzburg-Landau potential, which has quartic growth (i.e. $\left.\mathcal{W}(r)=\mathcal{O}\left(r^{4}\right)\right)$ by

$$
\mathcal{W}(r)= \begin{cases}(r-1)^{2}, & r>1, \\ \frac{1}{4}\left(r^{2}-1\right)^{2}, & r \in[-1,1], \\ (r+1)^{2}, & r<-1 .\end{cases}
$$

This is done because this function has the same behavior when $\phi \approx \pm 1$ and

$$
\sup _{r \in \mathbb{R}}\left|\mathcal{W}^{\prime \prime}(r)\right| \leq 2 .
$$

Moreover, as it has been shown in [8], limiting the growth of the potential to quadratic allows, in the continuous case, to obtain uniform $L^{\infty}$ estimates for the solution of Cahn Hilliard equations.

Next, we introduce a time step $\Delta t>0$. The scheme begins with a standard initialization step, i.e. $\phi^{0}=\left.\phi\right|_{t=0}$, $\mathbf{u}^{0}=\left.\mathbf{u}\right|_{t=0}$ and $p^{0}=\left.\mathbf{p}\right|_{t=0}$. Then, for $k \geq 0$, we proceed as follows:

Phase field and chemical potential: Find $\phi^{k+1}$ and $\mu^{k+1}$ that solve:

$$
\begin{cases}\frac{\phi^{k+1}-\phi^{k}}{\Delta t}+\mathbf{u}^{k+1} \cdot \nabla \phi^{k}=\nabla \cdot\left(M\left(\phi^{k}\right) \nabla \mu^{k+1}\right), & \text { in } \Omega, \\ \mu^{k+1}=\frac{\gamma}{\delta}\left[\mathcal{W}^{\prime}\left(\phi^{k}\right)+\mathcal{A}\left(\phi^{k+1}-\phi^{k}\right)\right]-\gamma \delta \Delta \phi^{k+1}+\frac{1}{2} \rho^{\prime}\left(\phi^{k}\right) \mathbf{u}^{k} \cdot \mathbf{u}^{k+1}, & \text { in } \Omega, \\ \alpha\left(\frac{\phi^{k+1}-\phi^{k}}{\Delta t}+\mathbf{u}_{\boldsymbol{\tau}}^{k+1} \partial_{\boldsymbol{\tau}} \phi^{k}\right)+L\left(\phi^{k+1}, \phi^{k}\right)=0, & \text { on } \Gamma \\ M\left(\phi^{k}\right) \partial_{\mathbf{n}} \mu^{k+1}=0, & \text { on } \Gamma\end{cases}
$$

where $L(\Phi, \Psi):=\gamma\left(\delta \partial_{\mathbf{n}} \Phi+\Theta_{f s}^{\prime}(\Psi)+\mathcal{B}(\Phi-\Psi)\right)$. The values of $\mathcal{A}$ and $\mathcal{B}$ will be defined below.

Auxiliary variables: Define

$$
\hat{\phi}(x)=\left\{\begin{array}{ll}
\phi^{k+1}(x), & \left|\phi^{k+1}(x)\right| \leq 1, \\
\operatorname{sgn}\left(\phi^{k+1}(x)\right), & \left|\phi^{k+1}(x)\right|>1,
\end{array} \quad x \in \Omega,\right.
$$

and $\rho^{k+1}=\rho(\hat{\phi})$. Define also

$$
\rho^{\star}=\frac{1}{2}\left(\rho^{k+1}+\rho^{k}\right), \quad p^{\sharp}=2 p^{k}-p^{k-1} .
$$

Velocity: Find $\mathbf{u}^{k+1}$ that solves

$$
\begin{cases}\frac{\rho^{\star} \mathbf{u}^{k+1}-\rho^{k} \mathbf{u}^{k}}{\Delta t}+\left(\rho^{k} \mathbf{u}^{k} \cdot \nabla\right) \mathbf{u}^{k+1}+\frac{1}{2} \nabla \cdot\left(\rho^{k} \mathbf{u}^{k}\right) \mathbf{u}^{k+1}-\nabla \cdot\left(\eta\left(\phi^{k+1}\right) \mathbf{T}\left(\mathbf{u}^{k+1}\right)\right) & \\ \quad+\nabla p^{\sharp}=\rho^{k+1} \mathbf{f}^{k+1}+\mu^{k+1} \nabla \phi^{k}+\frac{1}{2} \rho^{\prime}\left(\phi^{k}\right) \frac{\phi^{k+1}-\phi^{k}}{\Delta t} \mathbf{u}^{k}, & \text { in } \Omega, \\ \mathbf{u}_{\mathbf{n}}^{k+1}=0, & \text { on } \Gamma, \\ \beta\left(\phi^{k+1}\right)\left(\mathbf{u}_{\boldsymbol{\tau}}^{k+1}-\mathbf{U}_{\boldsymbol{\tau}}\right)+\eta\left(\phi^{k+1}\right) \mathbf{T}\left(\mathbf{u}^{k+1}\right)_{\mathbf{n} \boldsymbol{\tau}} & \text { on } \Gamma . \\ +\alpha\left(\frac{\phi^{k+1}-\phi^{k}}{\Delta t}+\mathbf{u}_{\boldsymbol{\tau}}^{k+1} \partial_{\boldsymbol{\tau}} \phi^{k}\right) \partial_{\boldsymbol{\tau}} \phi^{k}=0, & \end{cases}
$$


Penalization: The new pressure is computed by solving:

$$
\begin{cases}-\Delta\left(p^{k+1}-p^{k}\right)=-\frac{\varrho}{\Delta t} \nabla \cdot \mathbf{u}^{k+1}, & \text { in } \Omega \\ \partial_{\mathbf{n}}\left(p^{k+1}-p^{k}\right)=0, & \text { on } \Gamma\end{cases}
$$

where $\varrho:=\min \left\{\rho_{1}, \rho_{2}\right\}$.

Remark 4.1 (Discretization). Several comments are in order:

- The equations for the phase field and the velocity are weakly coupled, i.e. the only terms that couple these two equations are $\frac{1}{2} \rho^{\prime}\left(\phi^{k}\right) \mathbf{u}^{k} \cdot \mathbf{u}^{k+1}, \mathbf{u}^{k+1} \cdot \nabla \phi^{k}$ and its boundary analogue $\mathbf{u}_{\boldsymbol{\tau}}^{k+1} \partial_{\boldsymbol{\tau}} \phi^{k}$. In practice, it is possible to replace them with $\frac{1}{2} \rho^{\prime}\left(\phi^{k}\right)\left|\mathbf{u}^{k}\right|^{2}, \mathbf{u}^{k} \cdot \nabla \phi^{k}$ and $\mathbf{u}_{\boldsymbol{\tau}}^{k} \partial_{\boldsymbol{\tau}} \phi^{k}$, respectively. This would lead to a decoupled formulation which we conjecture would require a CFL-like condition for stability. For the analysis of the scheme we keep these two equations coupled. This coupling can be implemented, e.g., by using a fixed point iterative scheme.

- We have added stabilizing terms $\mathcal{A}\left(\phi^{k+1}-\phi^{k}\right)$ and $\mathcal{B}\left(\phi^{k+1}-\phi^{k}\right)$ to the chemical potential equation and boundary condition, respectively. These terms, formally, introduce an additional consistency error of order $\mathcal{A} \Delta t \phi_{t}$ and $\mathcal{B} \Delta t \phi_{t}$ which are, respectively, of the same order as the error introduced by the explicit treatment of the terms $\mathcal{W}^{\prime}(\phi)$ and $\Theta_{f s}^{\prime}(\phi)$. The reader is referred to [35], where a similar stabilization term is added to the chemical potential. This choice of stabilization parameters is nothing but a special case of the so-called convexity splitting schemes, see [42].

- The decoupling of the velocity and pressure is obtained using a fractional time stepping technique based on penalization of the divergence, as it was originally introduced in [19].

- By construction, the following bounds hold

$$
\varrho \leq \rho^{k+1} \leq \boldsymbol{\rho}, \quad \forall k \geq 0,
$$

with $\boldsymbol{\rho}=\max \left\{\rho_{1}, \rho_{2}\right\}$ and $\varrho:=\min \left\{\rho_{1}, \rho_{2}\right\}$.

The discrete energy law that this scheme satisfies is stated in the following:

Theorem 4.2 (Stability). Let $\mathbf{f} \equiv 0$ and $\mathbf{U} \equiv 0$. Assume that

$$
\frac{1}{2} \sup _{r \in \mathbb{R}}\left|\mathcal{W}^{\prime \prime}(r)\right| \leq \mathcal{A}, \quad \frac{1}{2} \sup _{r \in \mathbb{R}}\left|\Theta_{f s}^{\prime \prime}(r)\right| \leq \mathcal{B} .
$$

Then, the solution $\left\{\phi^{k}, \mu^{k}, \mathbf{u}^{k}, p^{k}\right\}_{k \geq 0}$ of scheme (4.2)-(4.3) and (4.4) satisfies the following discrete energy law,

$$
\begin{gathered}
\left\|\sigma^{k+1} \mathbf{u}^{k+1}\right\|_{\mathbf{L}^{2}}^{2}+2 \Delta t\left\|\sqrt{\eta\left(\phi^{k+1}\right)} \mathbf{T}\left(\mathbf{u}^{k+1}\right)\right\|_{\mathbf{L}^{2}}^{2}+\frac{\Delta t^{2}}{\varrho}\left\|\nabla p^{k+1}\right\|_{\mathbf{L}^{2}}^{2}+\frac{\Delta t^{2}}{\varrho}\left\|\nabla\left(p^{k}-p^{k-1}\right)\right\|_{\mathbf{L}^{2}}^{2} \\
+2 \Delta t\left\|\sqrt{\beta\left(\phi^{k+1}\right)} \mathbf{u}_{\boldsymbol{\tau}}^{k+1}\right\|_{\mathbf{L}^{2}(\Gamma)}^{2}+2 \Delta t\left\|\sqrt{M\left(\phi^{k}\right)} \nabla \mu^{k+1}\right\|_{L^{2}}^{2}+\gamma \delta\left\|\nabla \phi^{k+1}\right\|_{\mathbf{L}^{2}}^{2}+\gamma \delta\left\|\nabla\left(\phi^{k+1}-\phi^{k}\right)\right\|_{\mathbf{L}^{2}}^{2} \\
+\frac{2 \gamma}{\delta} \int_{\Omega} \mathcal{W}\left(\phi^{k+1}\right)+2 \alpha \Delta t\left\|\frac{\phi^{k+1}-\phi^{k}}{\Delta t}+\mathbf{u}_{\boldsymbol{\tau}}^{k+1} \partial_{\boldsymbol{\tau}} \phi^{k}\right\|_{L^{2}(\Gamma)}^{2}+2 \gamma \int_{\Gamma} \Theta_{f s}\left(\phi^{k+1}\right) \\
\leq\left\|\sigma^{k} \mathbf{u}^{k}\right\|_{\mathbf{L}^{2}}^{2}+\frac{\Delta t^{2}}{\varrho}\left\|\nabla p^{k}\right\|_{\mathbf{L}^{2}}^{2}+\gamma \delta\left\|\nabla \phi^{k}\right\|_{\mathbf{L}^{2}}^{2}+\frac{2 \gamma}{\delta} \int_{\Omega} \mathcal{W}\left(\phi^{k}\right)+2 \gamma \int_{\Gamma} \Theta_{f s}\left(\phi^{k}\right),
\end{gathered}
$$

where $\sigma^{k}:=\sqrt{\rho^{k}}$. 
Proof. We proceed as in the proof of the continuous case, i.e. Theorem 3.1. Multiply the discrete momentum equation (4.3) by $2 \Delta t \mathbf{u}^{k+1}$ and integrate. As it was noted in [19],

$$
2\left(\rho^{\star}\left|\mathbf{u}^{k+1}\right|^{2}-\rho^{k} \mathbf{u}^{k} \cdot \mathbf{u}^{k+1}\right)=\left|\sigma^{k+1} \mathbf{u}^{k+1}\right|^{2}-\left|\sigma^{k} \mathbf{u}^{k}\right|^{2}+\left|\sigma^{k}\left(\mathbf{u}^{k+1}-\mathbf{u}^{k}\right)\right|^{2} .
$$

Using integration by parts and the generalized Navier boundary condition,

$$
\begin{aligned}
-2 \Delta t \int_{\Omega} \nabla \cdot\left(\eta\left(\phi^{k+1}\right) \mathbf{T}\left(\mathbf{u}^{k+1}\right)\right) \cdot \mathbf{u}^{k+1}= & 2 \Delta t \int_{\Gamma} \beta\left(\phi^{k+1}\right)\left|\mathbf{u}_{\boldsymbol{\tau}}^{k+1}\right|^{2} \\
& +2 \alpha \Delta t \int_{\Gamma}\left(\frac{\phi^{k+1}-\phi^{k}}{\Delta t}+\mathbf{u}_{\boldsymbol{\tau}}^{k+1} \partial_{\boldsymbol{\tau}} \phi^{k}\right) \mathbf{u}_{\boldsymbol{\tau}}^{k+1} \partial_{\boldsymbol{\tau}} \phi^{k} \\
& +2 \Delta t\left\|\sqrt{\eta\left(\phi^{k+1}\right)} \mathbf{T}\left(\mathbf{u}^{k+1}\right)\right\|_{\mathbf{L}^{2}}^{2} .
\end{aligned}
$$

The convective term is treated using (2.5).

Finally, the term $\int_{\Omega} \nabla p^{\sharp} \cdot \mathbf{u}^{k+1}$, is handled using the penalization equation as in [19,35,37]. Notice that, these works are concerned with a no-slip boundary condition on the velocity. However, all that is needed for their arguments to hold is an impermeability condition on the velocity $(i . e . \mathbf{u} \cdot \mathbf{n}=0)$. For completeness, we repeat the arguments. To begin with, notice that

$$
-\int_{\Omega} \nabla p^{\sharp} \cdot \mathbf{u}^{k+1}=\int_{\Omega} \nabla\left(p^{k+1}-2 p^{k}+p^{k-1}\right) \cdot \mathbf{u}^{k+1}-\int_{\Omega} \nabla p^{k+1} \cdot \mathbf{u}^{k+1} .
$$

Multiply (4.4) by $2 \frac{\Delta t^{2}}{\varrho}\left(p^{k+1}-2 p^{k}+p^{k-1}\right)$ and integrate. After integration by parts we apply the identity $2 a(a-b)=a^{2}-b^{2}+(a-b)^{2}$ to obtain

$$
\begin{aligned}
-\frac{\Delta t^{2}}{\varrho}\left[\left\|\nabla\left(p^{k+1}-p^{k}\right)\right\|_{\mathbf{L}^{2}}^{2}-\left\|\nabla\left(p^{k}-p^{k-1}\right)\right\|_{\mathbf{L}^{2}}^{2}+\left\|\nabla\left(p^{k+1}-2 p^{k}+p^{k-1}\right)\right\|_{\mathbf{L}^{2}}^{2}\right] & \\
& +2 \Delta t \int_{\Omega} \mathbf{u}^{k+1} \cdot \nabla\left(p^{k+1}-2 p^{k}+p^{k-1}\right)=0 .
\end{aligned}
$$

Multiply (4.4) by $2 \frac{\Delta t^{2}}{\varrho} p^{k+1}$ and integrate. Using the identity mentioned before we get

$$
\frac{\Delta t^{2}}{\varrho}\left[\left\|\nabla p^{k+1}\right\|_{\mathbf{L}^{2}}^{2}-\left\|\nabla p^{k}\right\|_{\mathbf{L}^{2}}^{2}+\left\|\nabla\left(p^{k+1}-p^{k}\right)\right\|_{\mathbf{L}^{2}}^{2}\right]=2 \Delta t \int_{\Omega} \mathbf{u}^{k+1} \cdot \nabla p^{k+1} .
$$

Take the difference between (4.4) at time steps $k+1$ and $k$ and multiply the result by $p^{k+1}-2 p^{k}+p^{k-1}$. An application of the Cauchy Schwarz inequality and the lower bound of (4.5) allow us to obtain the following estimate

$$
\frac{\Delta t^{2}}{\varrho}\left\|\nabla\left(p^{k+1}-2 p^{k}+p^{k-1}\right)\right\|_{\mathbf{L}^{2}}^{2} \leq \varrho\left\|\mathbf{u}^{k+1}-\mathbf{u}^{k}\right\|_{\mathbf{L}^{2}}^{2} \leq\left\|\sigma^{k}\left(\mathbf{u}^{k+1}-\mathbf{u}^{k}\right)\right\|_{\mathbf{L}^{2}}^{2} ;
$$

see [19] for details.

From the observations above, we arrive at

$$
\begin{aligned}
\left\|\sigma^{k+1} \mathbf{u}^{k+1}\right\|_{\mathbf{L}^{2}}^{2}+ & 2 \Delta t\left\|\sqrt{\eta\left(\phi^{k+1}\right)} \mathbf{T}\left(\mathbf{u}^{k+1}\right)\right\|_{\mathbf{L}^{2}}^{2}+\frac{\Delta t^{2}}{\varrho}\left\|\nabla p^{k+1}\right\|_{\mathbf{L}^{2}}^{2}+\frac{\Delta t^{2}}{\varrho}\left\|\nabla\left(p^{k}-p^{k-1}\right)\right\|_{\mathbf{L}^{2}}^{2} \\
& +2 \Delta t\left\|\sqrt{\beta\left(\phi^{k+1}\right)} \mathbf{u}_{\boldsymbol{\tau}}^{k+1}\right\|_{\mathbf{L}^{2}(\Gamma)}^{2}+2 \alpha \Delta t \int_{\Gamma}\left(\frac{\phi^{k+1}-\phi^{k}}{\Delta t}+\mathbf{u}_{\boldsymbol{\tau}}^{k+1} \partial_{\boldsymbol{\tau}} \phi^{k}\right) \mathbf{u}_{\boldsymbol{\tau}}^{k+1} \partial_{\boldsymbol{\tau}} \phi^{k} \\
& \leq 2 \Delta t \int_{\Omega} \mu^{k+1} \nabla \phi^{k} \cdot \mathbf{u}^{k+1}+\int_{\Omega} \rho^{\prime}\left(\phi^{k}\right)\left(\phi^{k+1}-\phi^{k}\right) \mathbf{u}^{k} \cdot \mathbf{u}^{k+1}+\left\|\sigma^{k} \mathbf{u}^{k}\right\|_{\mathbf{L}^{2}}^{2}+\frac{\Delta t^{2}}{\varrho}\left\|\nabla p^{k}\right\|_{\mathbf{L}^{2}}^{2} .
\end{aligned}
$$


We multiply the phase field equation (first equation of (4.2)) by $2 \Delta t \mu^{k+1}$ and integrate over $\Omega$. Using the impermeability condition $M\left(\phi^{k}\right) \partial_{\mathbf{n}} \mu^{k+1}=0$ we obtain

$$
2 \int_{\Omega}\left(\phi^{k+1}-\phi^{k}\right) \mu^{k+1}+2 \Delta t \int_{\Omega} \mu^{k+1} \mathbf{u}^{k+1} \cdot \nabla \phi^{k}=-2 \Delta t\left\|\sqrt{M\left(\phi^{k}\right)} \nabla \mu^{k+1}\right\|_{\mathbf{L}^{2}}^{2} .
$$

Multiply the equation for the chemical potential (second equation of $(4.2))$ by $-2\left(\phi^{k+1}-\phi^{k}\right.$ ) and integrate over $\Omega$

$$
\begin{aligned}
-2 \int_{\Omega}\left(\phi^{k+1}-\phi^{k}\right) \mu^{k+1}= & 2 \gamma \delta \int_{\Omega} \Delta \phi^{k+1}\left(\phi^{k+1}-\phi^{k}\right) \\
& -2 \frac{\gamma}{\delta}\left(\int_{\Omega} \mathcal{W}^{\prime}\left(\phi^{k}\right)\left(\phi^{k+1}-\phi^{k}\right)+\mathcal{A}\left\|\phi^{k+1}-\phi^{k}\right\|_{L^{2}}^{2}\right)-\int_{\Omega} \rho^{\prime}\left(\phi^{k}\right)\left(\phi^{k+1}-\phi^{k}\right) \mathbf{u}^{k} \cdot \mathbf{u}^{k+1},
\end{aligned}
$$

adding these two relations we obtain

$$
\begin{aligned}
-2 \gamma \delta \int_{\Omega} \Delta \phi^{k+1} & \left(\phi^{k+1}-\phi^{k}\right)+2 \frac{\gamma}{\delta}\left(\int_{\Omega} \mathcal{W}^{\prime}\left(\phi^{k}\right)\left(\phi^{k+1}-\phi^{k}\right)+\mathcal{A}\left\|\phi^{k+1}-\phi^{k}\right\|_{L^{2}}^{2}\right) \\
& +2 \Delta t\left\|\sqrt{M\left(\phi^{k}\right)} \nabla \mu^{k+1}\right\|_{\mathbf{L}^{2}}^{2}+2 \Delta t \int_{\Omega} \mu^{k+1} \mathbf{u}^{k+1} \cdot \nabla \phi^{k}=-\int_{\Omega} \rho^{\prime}\left(\phi^{k}\right)\left(\phi^{k+1}-\phi^{k}\right) \mathbf{u}^{k} \cdot \mathbf{u}^{k+1} .
\end{aligned}
$$

Integrate by parts the first term on the left hand side of (4.8) and use the identity $2 a(a-b)=a^{2}-b^{2}+(a-b)^{2}$ to get

$2 \gamma \delta \int_{\Omega} \Delta \phi^{k+1}\left(\phi^{k+1}-\phi^{k}\right)=2 \gamma \delta \int_{\Gamma} \partial_{\mathbf{n}} \phi^{k+1}\left(\phi^{k+1}-\phi^{k}\right)-\gamma \delta\left[\left\|\nabla \phi^{k+1}\right\|_{\mathbf{L}^{2}}^{2}-\left\|\nabla \phi^{k}\right\|_{\mathbf{L}^{2}}^{2}+\left\|\nabla\left(\phi^{k+1}-\phi^{k}\right)\right\|_{\mathbf{L}^{2}}^{2}\right]$.

The integral involving the derivative of the Ginzburg Landau potential is treated, as in [37], using a Taylor expansion,

$$
\mathcal{W}\left(\phi^{k+1}\right)-\mathcal{W}\left(\phi^{k}\right)=\mathcal{W}^{\prime}\left(\phi^{k}\right)\left(\phi^{k+1}-\phi^{k}\right)+\frac{\mathcal{W}^{\prime \prime}\left(\varphi_{1}^{k}\right)}{2}\left(\phi^{k+1}-\phi^{k}\right)^{2},
$$

for some $\varphi_{1}^{k}$. From these observations (4.8) can be equivalently rewritten as

$$
\begin{aligned}
2 \Delta t \int_{\Omega} \mu^{k+1} \mathbf{u}^{k+1} \nabla \phi^{k} & +2 \Delta t\left\|\sqrt{M\left(\phi^{k}\right)} \nabla \mu^{k+1}\right\|_{\mathbf{L}^{2}}^{2}+\gamma \delta\left\|\nabla \phi^{k+1}\right\|_{\mathbf{L}^{2}}^{2}+\gamma \delta\left\|\nabla\left(\phi^{k+1}-\phi^{k}\right)\right\|_{\mathbf{L}^{2}}^{2} \\
+ & 2 \frac{\gamma}{\delta} \int_{\Omega} \mathcal{W}\left(\phi^{k+1}\right)+2 \frac{\gamma}{\delta} \int_{\Omega}\left(\mathcal{A}-\frac{\mathcal{W}^{\prime \prime}\left(\varphi_{1}^{k}\right)}{2}\right)\left(\phi^{k+1}-\phi^{k}\right)^{2}=\gamma \delta\left\|\nabla \phi^{k}\right\|_{\mathbf{L}^{2}}^{2} \\
& +2 \frac{\gamma}{\delta} \int_{\Omega} \mathcal{W}\left(\phi^{k}\right)+2 \gamma \delta \int_{\Gamma} \partial_{\mathbf{n}} \phi^{k+1}\left(\phi^{k+1}-\phi^{k}\right)-\int_{\Omega} \rho^{\prime}\left(\phi^{k}\right)\left(\phi^{k+1}-\phi^{k}\right) \mathbf{u}^{k} \cdot \mathbf{u}^{k+1} .
\end{aligned}
$$

Adding (4.7) and (4.9) we obtain

$$
\begin{aligned}
\left\|\sigma^{k+1} \mathbf{u}^{k+1}\right\|_{\mathbf{L}^{2}}^{2}+2 \Delta t & \left\|\sqrt{\eta\left(\phi^{k+1}\right)} \mathbf{T}\left(\mathbf{u}^{k+1}\right)\right\|_{\mathbf{L}^{2}}^{2}+\frac{\Delta t^{2}}{\varrho}\left\|\nabla p^{k+1}\right\|_{\mathbf{L}^{2}}^{2}+\frac{\Delta t^{2}}{\varrho}\left\|\nabla\left(p^{k}-p^{k-1}\right)\right\|_{\mathbf{L}^{2}}^{2} \\
& +2 \Delta t\left\|\sqrt{M\left(\phi^{k}\right)} \nabla \mu^{k+1}\right\|_{\mathbf{L}^{2}}^{2}+\gamma \delta\left\|\nabla \phi^{k+1}\right\|_{\mathbf{L}^{2}}^{2}+\gamma \delta\left\|\nabla\left(\phi^{k+1}-\phi^{k}\right)\right\|_{\mathbf{L}^{2}}^{2} \\
+ & 2 \frac{\gamma}{\delta} \int_{\Omega} \mathcal{W}\left(\phi^{k+1}\right)+2 \Delta t\left\|\sqrt{\beta\left(\phi^{k+1}\right)} \mathbf{u}_{\boldsymbol{\tau}}^{k+1}\right\|_{\mathbf{L}^{2}(\Gamma)}^{2}+2 \frac{\gamma}{\delta} \int_{\Omega}\left(\mathcal{A}-\frac{\mathcal{W}^{\prime \prime}\left(\varphi_{1}^{k}\right)}{2}\right)\left(\phi^{k+1}-\phi^{k}\right)^{2} \\
+ & 2 \alpha \Delta t \int_{\Gamma}\left(\frac{\phi^{k+1}-\phi^{k}}{\Delta t}+\mathbf{u}_{\boldsymbol{\tau}}^{k+1} \partial_{\boldsymbol{\tau}} \phi^{k}\right) \mathbf{u}_{\boldsymbol{\tau}}^{k+1} \partial_{\boldsymbol{\tau}} \phi^{k} \leq\left\|\sigma^{k} \mathbf{u}^{k}\right\|_{\mathbf{L}^{2}}^{2}+\frac{\Delta t^{2}}{\varrho}\left\|\nabla p^{k}\right\|_{\mathbf{L}^{2}}^{2}+\gamma \delta\left\|\nabla \phi^{k}\right\|_{\mathbf{L}^{2}}^{2} \\
& +2 \frac{\gamma}{\delta} \int_{\Omega} \mathcal{W}\left(\phi^{k}\right)+2 \gamma \delta \int_{\Gamma} \partial_{\mathbf{n}} \phi^{k+1}\left(\phi^{k+1}-\phi^{k}\right) .
\end{aligned}
$$


Using the boundary condition on the phase variable (third equation of (4.2)), we can rewrite the last term on the right hand side of this inequality as follows:

$$
-\gamma \delta \partial_{\mathbf{n}} \phi^{k+1}=\alpha\left(\frac{\phi^{k+1}-\phi^{k}}{\Delta t}+\mathbf{u}_{\tau}^{k+1} \partial_{\boldsymbol{\tau}} \phi^{k}\right)+\gamma\left(\Theta_{f s}^{\prime}\left(\phi^{k}\right)+\mathcal{B}\left(\phi^{k+1}-\phi^{k}\right)\right),
$$

Using a Taylor expansion for $\Theta_{f s}^{\prime}$ we then arrive at

$$
\begin{gathered}
\left\|\sigma^{k+1} \mathbf{u}^{k+1}\right\|_{\mathbf{L}^{2}}^{2}+2 \Delta t\left\|\sqrt{\eta\left(\phi^{k+1}\right)} \mathbf{T}\left(\mathbf{u}^{k+1}\right)\right\|_{\mathbf{L}^{2}}^{2}+\frac{\Delta t^{2}}{\varrho}\left\|\nabla p^{k+1}\right\|_{\mathbf{L}^{2}}^{2}+\frac{\Delta t^{2}}{\varrho}\left\|\nabla\left(p^{k}-p^{k-1}\right)\right\|_{\mathbf{L}^{2}}^{2} \\
+2 \Delta t\left\|\sqrt{M\left(\phi^{k}\right)} \nabla \mu^{k+1}\right\|_{\mathbf{L}^{2}}^{2}+\gamma \delta\left\|\nabla \phi^{k+1}\right\|_{\mathbf{L}^{2}}^{2}+\gamma \delta\left\|\nabla\left(\phi^{k+1}-\phi^{k}\right)\right\|_{\mathbf{L}^{2}}^{2} \\
+2 \frac{\gamma}{\delta} \int_{\Omega} \mathcal{W}\left(\phi^{k+1}\right)+2 \Delta t\left\|\sqrt{\beta\left(\phi^{k+1}\right)} \mathbf{u}_{\boldsymbol{\tau}}^{k+1}\right\|_{\mathbf{L}^{2}(\Gamma)}^{2}+2 \alpha \Delta t\left\|\frac{\phi^{k+1}-\phi^{k}}{\Delta t}+\mathbf{u}_{\boldsymbol{\tau}}^{k+1} \partial_{\boldsymbol{\tau}} \phi^{k}\right\|_{L^{2}(\Gamma)}^{2} \\
+2 \gamma \int_{\Gamma} \Theta_{f s}\left(\phi^{k+1}\right)+2 \gamma \int_{\Gamma}\left(\mathcal{B}-\frac{\Theta_{f s}^{\prime \prime}\left(\varphi_{2}^{k}\right)}{2}\right)\left(\phi^{k+1}-\phi^{k}\right)^{2}+2 \frac{\gamma}{\delta} \int_{\Omega}\left(\mathcal{A}-\frac{\mathcal{W}^{\prime \prime}\left(\varphi_{1}^{k}\right)}{2}\right)\left(\phi^{k+1}-\phi^{k}\right)^{2} \\
\leq\left\|\sigma^{k} \mathbf{u}^{k}\right\|_{\mathbf{L}^{2}}^{2}+\frac{\Delta t^{2}}{\varrho}\left\|\nabla p^{k}\right\|_{\mathbf{L}^{2}}^{2}+\gamma \delta\left\|\nabla \phi^{k}\right\|_{\mathbf{L}^{2}}^{2}+2 \frac{\gamma}{\delta} \int_{\Omega} \mathcal{W}\left(\phi^{k}\right)+2 \gamma \int_{\Gamma} \Theta_{f s}\left(\phi^{k}\right)
\end{gathered}
$$

Since, by assumption

$$
\mathcal{A} \geq \frac{1}{2}\left|\mathcal{W}^{\prime \prime}\left(\varphi_{1}^{k}\right)\right|, \quad \mathcal{B} \geq \frac{1}{2}\left|\Theta_{f s}^{\prime \prime}\left(\varphi_{2}^{k}\right)\right|
$$

we obtain the result.

Remark 4.3 (Stabilization parameters). Notice that, in Theorem 4.2, the conditions on the stabilization parameters $\mathcal{A}$ and $\mathcal{B}$ do not depend on the time step $\Delta t$. Given the definitions of $\mathcal{W}$ and $\Theta_{f s}$, the following inequalities hold:

$$
\mathcal{A} \geq 1, \quad \mathcal{B} \geq \frac{\pi^{2}}{2}
$$

The restriction imposed on $\mathcal{A}$ for stability is similar to the one obtained in previous works. See, for example, [35, 36]. As discussed in there, it is also possible to obtain conditionally stable schemes for $\mathcal{A}=0$. However, the time-step restriction is quite severe, e.g. $\Delta t=\mathcal{O}\left(\delta^{4}\right)$. In the numerical experiments of Section 6 we set the stabilization parameters as small as their constraints allow.

\section{SPACE Discretization}

In this section we introduce a fully discrete version of the above described splitting algorithm. To this end, let $h>0$ be a space discretization parameter. We introduce finite dimensional spaces $W_{h} \subset H^{1}(\Omega), \mathbf{X}_{h} \subset \mathbf{V}$, where

$$
\mathbf{V}:=\left\{\mathbf{v} \in \mathbf{H}^{1}(\Omega):\left.\mathbf{v} \cdot \mathbf{n}\right|_{\Gamma}=0\right\},
$$

and $M_{h} \subset H_{f=0}^{1}(\Omega)$. The spaces $\mathbf{X}_{h}$ and $M_{h}$ are assumed to be compatible, in the sense that they satisfy the so-called discrete LBB condition. That is, there is a constant $c$ independent of $h$ such that

$$
\sup _{\mathbf{v}_{h} \in \mathbf{X}_{h}} \frac{\int_{\Omega} q_{h} \nabla \cdot \mathbf{v}_{h}}{\left\|\mathbf{v}_{h}\right\|_{\mathbf{H}^{1}}} \geq c\left\|q_{h}\right\|_{L^{2}}, \quad \forall q_{h} \in M_{h} .
$$

Examples of these spaces can be found, e.g. in $[7,12,16]$. 
To simplify notation, we shall denote by $\langle\cdot, \cdot\rangle$ the $L^{2}(\Omega)$-inner product and by $[\cdot, \cdot]$ the $L^{2}(\Gamma)$-inner product. Moreover, for any sequence $\left\{\varphi^{k}\right\}$

$$
\mathfrak{d} \varphi^{k+1}=\varphi^{k+1}-\varphi^{k}
$$

The algorithm begins with a standard initialization step, that is we set $\phi_{h}^{0} \in W_{h}, \mathbf{u}_{h}^{0} \in \mathbf{X}_{h}$ and $p_{h}^{0} \in M_{h}$ as suitable approximations to $\left.\phi\right|_{t=0},\left.\mathbf{u}\right|_{t=0}$ and $\left.\mathbf{p}\right|_{t=0}$, respectively. Then, for $k \geq 0$ we advance in several steps:

Phase field and chemical potential: Find $\phi_{h}^{k+1}, \mu_{h}^{k+1} \in W_{h}$ that solve

$$
\begin{gathered}
\left\langle\frac{\mathfrak{d} \phi_{h}^{k+1}}{\Delta t}, \psi_{h}\right\rangle+\left\langle\mathbf{u}_{h}^{k+1} \cdot \nabla \phi_{h}^{k}, \psi_{h}\right\rangle+\left\langle M\left(\phi_{h}^{k}\right) \nabla \mu_{h}^{k+1}, \nabla \psi_{h}\right\rangle=0, \quad \forall \psi_{h} \in W_{h}, \\
\left\langle\mu_{h}^{k+1}, \bar{\psi}_{h}\right\rangle=\gamma \delta\left\langle\nabla \phi_{h}^{k+1}, \nabla \bar{\psi}_{h}\right\rangle+\frac{\gamma}{\delta}\left\langle\mathcal{W}^{\prime}\left(\phi_{h}^{k}\right)+\mathcal{A} \mathfrak{d} \phi_{h}^{k+1}, \bar{\psi}_{h}\right\rangle+\frac{1}{2}\left\langle\rho^{\prime}\left(\phi_{h}^{k}\right) \mathbf{u}_{h}^{k} \cdot \mathbf{u}_{h}^{k+1}, \bar{\psi}_{h}\right\rangle \\
+\alpha\left[\frac{\mathfrak{d} \phi_{h}^{k+1}}{\Delta t}+\mathbf{u}_{h \boldsymbol{\tau}}^{k+1} \partial_{\boldsymbol{\tau}} \phi_{h}^{k}, \bar{\psi}_{h}\right]+\gamma\left[\Theta_{f s}^{\prime}\left(\phi_{h}^{k}\right)+\mathcal{B} \mathfrak{d} \phi_{h}^{k+1}, \bar{\psi}_{h}\right] \quad \forall \bar{\psi}_{h} \in W_{h} .
\end{gathered}
$$

Auxiliary variables: Define

$$
\hat{\phi}_{h}(x)=\left\{\begin{array}{ll}
\phi_{h}^{k+1}(x), & \left|\phi_{h}^{k+1}(x)\right| \leq 1, \\
\operatorname{sgn}\left(\phi_{h}^{k+1}(x)\right), & \left|\phi_{h}^{k+1}(x)\right|>1,
\end{array} \quad x \in \Omega,\right.
$$

and $\rho_{h}^{k+1}=\rho\left(\hat{\phi}_{h}\right)$. Define also

$$
\rho_{h}^{\star}=\frac{1}{2}\left(\rho_{h}^{k+1}+\rho_{h}^{k}\right), \quad p_{h}^{\sharp}=2 p_{h}^{k}-p_{h}^{k-1} .
$$

Velocity: Find $\mathbf{u}_{h}^{k+1} \in \mathbf{X}_{h}$ that solves

$$
\begin{aligned}
&\left\langle\frac{\rho_{h}^{\star} \mathbf{u}_{h}^{k+1}-\rho_{h}^{k} \mathbf{u}_{h}^{k}}{\Delta t}+\right.\left.\left(\rho_{h}^{k} \mathbf{u}_{h}^{k} \cdot \nabla\right) \mathbf{u}_{h}^{k+1}+\frac{1}{2} \nabla \cdot\left(\rho_{h}^{k} \mathbf{u}_{h}^{k}\right) \mathbf{u}_{h}^{k+1}, \mathbf{v}_{h}\right\rangle+\left\langle\eta\left(\phi_{h}^{k+1}\right) \mathbf{T}\left(\mathbf{u}_{h}^{k+1}\right), \mathbf{T}\left(\mathbf{v}_{h}\right)\right\rangle \\
&-\left\langle p_{h}^{\sharp}, \nabla \cdot \mathbf{v}_{h}\right\rangle+\left[\beta\left(\phi_{h}^{k+1}\right) \mathbf{u}_{h \boldsymbol{\tau}}^{k+1}, \mathbf{v}_{h \boldsymbol{\tau}}\right]+\alpha\left[\frac{\mathfrak{d} \phi_{h}^{k+1}}{\Delta t}+\mathbf{u}_{h}^{k+1} \partial_{\boldsymbol{\tau}} \phi_{h}^{k}, \mathbf{v}_{h \boldsymbol{\tau}} \partial_{\boldsymbol{\tau}} \phi_{h}^{k}\right]=\left\langle\rho_{h}^{k+1} \mathbf{f}^{k+1}, \mathbf{v}_{h}\right\rangle \\
&+\left\langle\mu_{h}^{k+1} \nabla \phi_{h}^{k}, \mathbf{v}_{h}\right\rangle+\frac{1}{2}\left\langle\rho^{\prime}\left(\phi_{h}^{k}\right) \frac{\mathfrak{d} \phi_{h}^{k+1}}{\Delta t} \mathbf{u}_{h}^{k}, \mathbf{v}_{h}\right\rangle+\left[\beta\left(\phi_{h}^{k+1}\right) \mathbf{U}_{\boldsymbol{\tau}}, \mathbf{v}_{h \boldsymbol{\tau}}\right], \quad \forall v_{h} \in \mathbf{X}_{h} .
\end{aligned}
$$

Penalization: The new pressure $p_{h}^{k+1}$ solves

$$
\left\langle\nabla \mathfrak{d} p_{h}^{k+1}, \nabla q_{h}\right\rangle=-\frac{\varrho}{\Delta t}\left\langle\nabla \cdot \mathbf{u}_{h}^{k+1}, q_{h}\right\rangle, \quad \forall q_{h} \in M_{h} .
$$


Corollary 5.1 (Fully discrete stability). Let $\mathbf{f} \equiv 0$ and $\mathbf{U} \equiv 0$. Under the conditions of Theorem 4.2, this scheme satisfies the following discrete energy law:

$$
\begin{array}{r}
\left\|\sigma_{h}^{k+1} \mathbf{u}_{h}^{k+1}\right\|_{\mathbf{L}^{2}}^{2}+2 \Delta t\left\|\sqrt{\eta\left(\phi_{h}^{k+1}\right)} \mathbf{T}\left(\mathbf{u}_{h}^{k+1}\right)\right\|_{\mathbf{L}^{2}}^{2}+2 \Delta t\left\|\sqrt{\beta\left(\phi_{h}^{k+1}\right)} \mathbf{u}_{h \boldsymbol{\tau}}^{k+1}\right\|_{\mathbf{L}^{2}(\Gamma)}^{2}+\frac{\Delta t^{2}}{\varrho}\left\|\nabla p_{h}^{k+1}\right\|_{\mathbf{L}^{2}}^{2} \\
+\frac{\Delta t^{2}}{\varrho}\left\|\nabla \mathfrak{d} p_{h}^{k}\right\|_{\mathbf{L}^{2}}^{2}+2 \Delta t\left\|\sqrt{M\left(\phi_{h}^{k}\right)} \nabla \mu_{h}^{k+1}\right\|_{\mathbf{L}^{2}}^{2}+\gamma \delta\left\|\nabla \phi_{h}^{k+1}\right\|_{\mathbf{L}^{2}}^{2}+\gamma \delta\left\|\nabla \mathfrak{d} \phi_{h}^{k+1}\right\|_{\mathbf{L}^{2}}^{2}+2 \frac{\gamma}{\delta} \int_{\Omega} \mathcal{W}\left(\phi_{h}^{k+1}\right) \\
+2 \gamma \int_{\Gamma} \Theta_{f s}\left(\phi_{h}^{k+1}\right)+2 \alpha \Delta t\left\|\frac{\mathfrak{d} \phi_{h}^{k+1}}{\Delta t}+\mathbf{u}_{h}^{k+1} \partial_{\boldsymbol{\tau}} \phi_{h}^{k}\right\|_{L^{2}(\Gamma)}^{2} \leq\left\|\sigma_{h}^{k} \mathbf{u}_{h}^{k}\right\|_{\mathbf{L}^{2}}^{2}+\frac{\Delta t^{2}}{\varrho}\left\|\nabla p_{h}^{k}\right\|_{\mathbf{L}^{2}}^{2}+\gamma \delta\left\|\nabla \phi_{h}^{k}\right\|_{\mathbf{L}^{2}}^{2} \\
+2 \frac{\gamma}{\delta} \int_{\Omega} \mathcal{W}\left(\phi_{h}^{k}\right)+2 \gamma \int_{\Gamma} \Theta_{f s}\left(\phi_{h}^{k}\right)
\end{array}
$$

Proof. Set $\mathbf{v}_{h}=2 \Delta t \mathbf{u}_{h}^{k+1}$ in (5.4), $\psi_{h}=2 \Delta t \mu_{h}^{k+1}$ in (5.1) and $\bar{\psi}_{h}=2 \mathfrak{d} \phi_{h}^{k+1}$ in (5.2), respectively. Repeat the steps used to obtain Theorem 4.2 .

The importance of the energy law stated in Corollary 5.1 is not only that the numerical scheme possesses desirable properties. In addition, this serves as a formal a priori estimate from which existence of solutions for problem (5.1)-(5.5) can be obtained. This is the content of the following:

Theorem 5.2 (Existence of discrete solutions). Under the setting of Corollary 5.1, the scheme (5.1)-(5.5) always has a solution. Moreover, any solution satisfies the discrete energy law (5.6).

Proof. Reference [29] provides a detailed proof of existence of fully discrete solutions for a more general model that includes the one presented here. Hence, we only sketch the steps. The main idea is to use the Leray-Schauder theorem [43]. To do so we, on the basis of (5.1)-(5.5), introduce a map $\left(\phi_{h}, \mu_{h}, \mathbf{u}_{h}, p_{h}\right) \mapsto\left(\hat{\phi}_{h}, \hat{\mu}_{h}, \hat{\mathbf{u}}_{h}, \hat{p}_{h}\right)$ such that any fixed point is, by construction, a solution to our problem. Simply put this map is obtained by doing the following replacements:

(5.1) $\phi_{h}^{k+1} \leftarrow \hat{\phi}_{h}, \mu_{h}^{k+1} \leftarrow \hat{\mu}_{h}$ and $\mathbf{u}_{h}^{k+1} \leftarrow \mathbf{u}_{h}$.

(5.2) $\mu_{h}^{k+1} \leftarrow \hat{\mu}_{h}, \mathbf{u}_{h}^{k+1} \leftarrow \mathbf{u}_{h}$. The variable $\phi_{h}^{k+1}$ is replaced by $\phi_{h}$ when it is multiplied by the stabilization parameters $\mathcal{A}$ and $\mathcal{B}$; in all other places it is replaced by $\hat{\phi}_{h}$.

(5.3) $\phi_{h}^{k+1} \leftarrow \phi_{h}$.

(5.4) All the variables at level $k+1$ that appear on the left hand side are replaced by their hatted counterpart, except when $\phi_{h}^{k+1}$ serves as argument for a material function. The ones on the right hand side by the unhatted one.

(5.5) $p_{h}^{k+1} \leftarrow \hat{p}_{h}$ and $\mathbf{u}_{h}^{k+1} \leftarrow \hat{\mathbf{u}}_{h}$.

This map is affine, hence it is well defined. The next step is to obtain bounds on this operator, which are obtained as in the proof of Corollary 5.1. This allows us to conclude. See [29] for details.

\section{NumericAl EXPERIMENTS}

We now illustrate the performance of the method developed in previous Sections in a series of numerical examples. All the computations have been performed with the help of the deal. II library $($ see $[2,3])$. The space discretization is obtained using $\mathbb{Q}_{2}$ elements for the phase variable and Taylor-Hood (i.e. $\left(\mathbb{Q}_{2}, \mathbb{Q}_{1}\right)$ ) for the velocity-pressure. In order to properly resolve the interfacial layer, the mesh is computed adaptively. The level of refinement and local mesh sizes will be indicated in each test case. The time step is set initially to $\Delta t=10^{-3}$ and it is reduced if a local CFL-like condition is violated i.e.

$$
\Delta t=\min \left\{10^{-3}, \frac{1}{2} \max _{K \in \mathcal{T}_{h}}\left\{\frac{h_{K}^{3 / 2}}{\left\|\mathbf{u}_{h}\right\|_{L^{\infty}(K)}}\right\}\right\} .
$$


The reason to impose such a restriction on the time step is in order to avoid having to solve the weakly coupled problem as explained in Remark 4.1.

\subsection{Symmetric Couette flow}

The first example is motivated by $[15,31]$. We consider a variant of a Couette flow for a two phase fluid. The geometry consists of the rectangle $\Omega=(-3,3) \times(0,1)$. A velocity $V \mathbf{e}_{x}$ (respectively $\left.-V \mathbf{e}_{x}\right)$ is imposed on the top (respectively bottom) sides of the rectangle. Notice that to obtain a true Couette flow periodicity conditions should have been imposed on these sides. However, due to implementation reasons we did not do this, instead we apply the generalized Navier boundary condition. The initial configuration of the fluid is such that the heavier fluid occupies $(-1,1) \times(0,1)$ and the lighter the rest of the domain. The fluid is initially at rest.

The results of $[15,31]$ are restricted to the matched density case. While certainly our method is capable of handling this, we prefer to show the effect of the variable density. The material parameters are the following: $\rho_{1} / \rho_{2}=10, \eta_{1}=\eta_{2}=10, \beta_{1}=\beta_{2}=1.5, M_{1}=M_{2}=0.01, \alpha=10^{-2}, \gamma=10$ and $\theta_{s}=90^{\circ}$. The shear velocity is $V=1.5$. The interface thickness is $2.5 \times 10^{-2}$. The local mesh size away from the interface is about 0.125 and near the interface $8 \times 10^{-3}$. The result for times $t=0,0.5,1,1.5,1.75,2$ is shown in Figure 1 . As expected, the higher density leads to smaller displacements due to the bigger inertia of the fluid. Moreover, since the surface tension is sufficiently big, the interface evolves from a flat surface to a curved one.

\subsection{Asymmetric Couette flow}

As a second example, we consider an asymmetric variant of the case of Section 6.1. In this case, however, $\beta_{2}=0.6, \gamma=1$ and $\theta_{s}=68^{\circ}$. The interface thickness is $2.5 \times 10^{-2}$. The evolution for times $0.5,1,1.5,1.75,2$ and 2.5 is shown in Figure 2. As we can see, the smaller, compared to Section 6.1, value of the surface tension is not sufficient to prevent a significant movement of the interface.

We also consider a different initial profile. The rest of the configuration is the same as in the previous case. The result is shown in Figure 3.

\subsection{Droplet relaxation}

The next test case takes inspiration from [26] and [10]. We consider the spreading of a droplet over a wall under the action of gravity and surface tension. We investigate the result of two competing effects: surface tension, which tends to keep the droplet round; and gravity, which flattens the droplet. In all the test-cases the material parameters are $\rho_{1}=1, \rho_{2}=100, \eta_{1}=10, \eta_{2}=1, \beta_{1}=\beta_{2}=1.5, \theta_{s}=68^{\circ}, M_{1}=M_{2}=10^{-2}$, and $\alpha=10^{-2}$. The interface thickness is $\delta=2.5 \times 10^{-2}$. The computational domain is $\Omega=(-1,1) \times(0,1)$ and the mesh is adaptively refined to resolve the transitional layer and it is such that the local mesh size near the interface is about $8 \times 10^{-3}$ and away from it $3 \times 10^{-2}$.

We first consider the case when the surface tension is small compared to gravity. To be precise, we set $\gamma=1$. Figure 4 shows the evolution of the interface of a droplet initially shaped like half a circle without gravity. Due to the difference between the initial angle and the static one (the uncompensated Young stress), the interface moves until the contact angle equals the static one, which happens at about time $t=3.3$. The evolution under the action of gravity is shown in Figure 5. As we can see, if gravity is present, the surface tension is not sufficient to preserve the shape of the droplet and it turns into a pool of heavier fluid on the bottom of the container.

To illustrate the opposite case, that is, when the surface tension effects are dominant, we set $\gamma=50$. Figure 6 shows the evolution of the interface without the action of gravity and Figure 7 under its action. In both cases a stationary shape is reached at about time $t=1.1$. As it is illustrated in Figure 8 the surface tension effects are so dominant that the final position of the interface is basically the same for both cases. Moreover, as it is also seen from Figure 8, the contact angle changes during evolution. Although this is a controversial issue, we just want to point out that this model allows for such effects. 

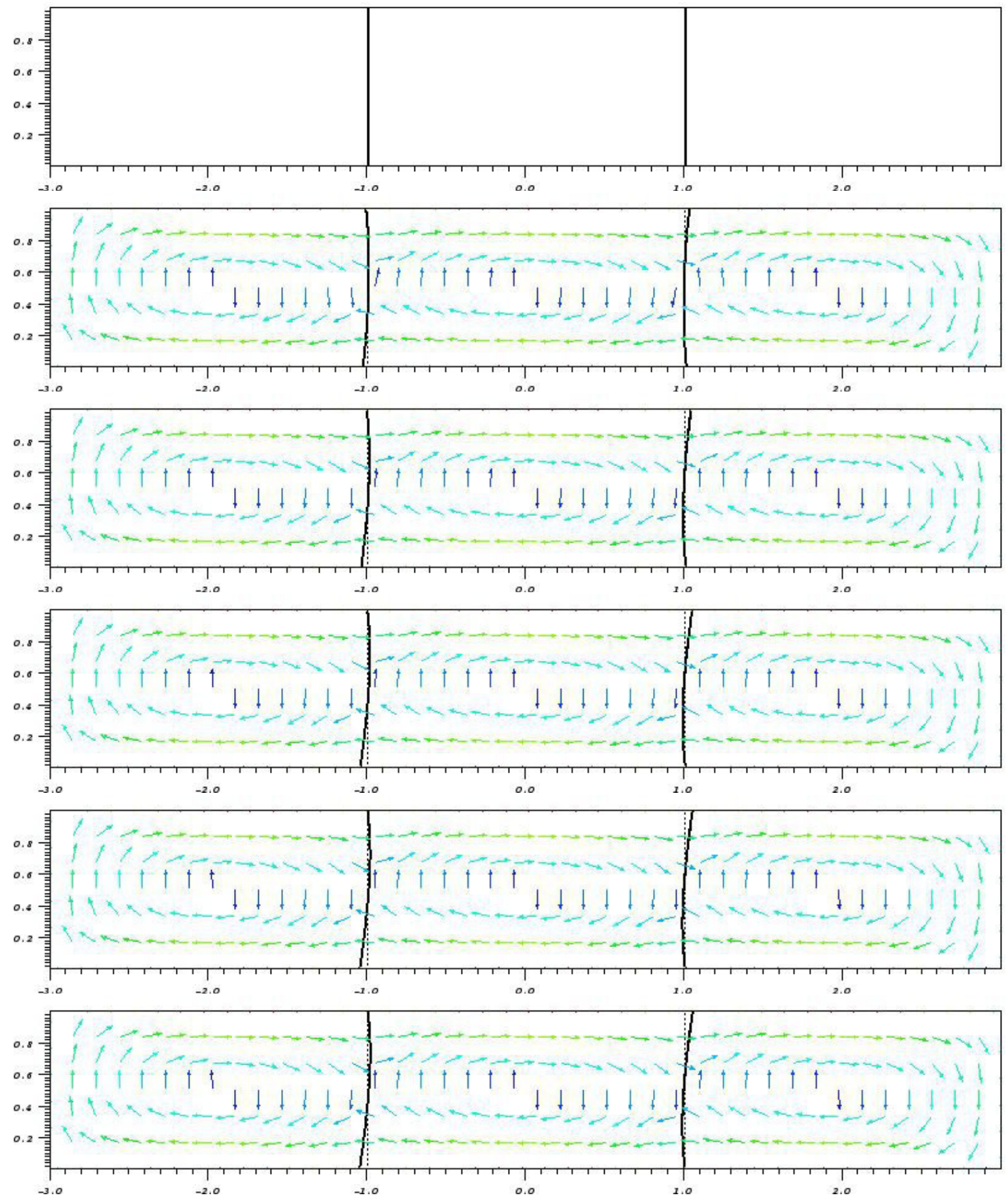

Figure 1. Symmetric Couette flow. The evolution of the interface is shown at times 0, 0.5, 1, 1.5, 1.75 and 2. The arrows represent the velocity. The dotted line is the initial position of the interface. The material parameters are: $\rho_{1} / \rho_{2}=10, \eta_{1}=\eta_{2}=10, \beta_{1}=\beta_{2}=1.5$, $M_{1}=M_{2}=0.01, \alpha=10^{-2}, \gamma=10$ and $\theta_{s}=90^{\circ}$ and $V=1.5$. The interface thickness is $2.5 \times 10^{-2}$. 

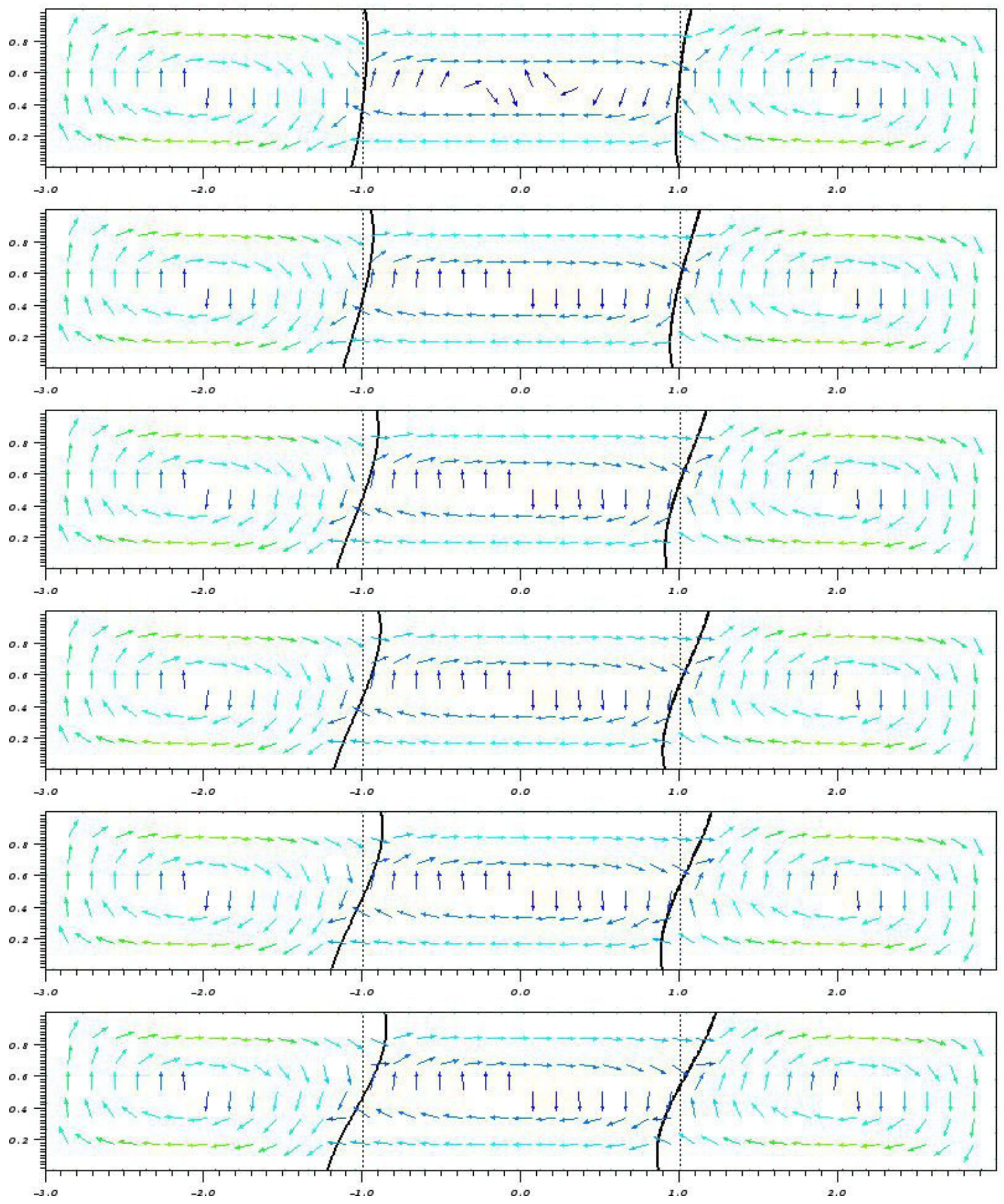

FIgURE 2. Asymmetric Couette flow with straight initial profile. The evolution of the interface is shown at times $0.5,1,1.5,1.75,2$ and 2.5 . The arrows represent the velocity. The dotted line is the initial position of the interface. The material parameters are $\rho_{1} / \rho_{2}=10, \eta_{1}=\eta_{2}=10$, $\beta_{1}=1.5, \beta_{2}=0.6, M_{1}=M_{2}=0.01, \alpha=10^{-2}, \gamma=1$ and $\theta_{s}=68^{\circ}$. The shear velocity is $V=1.5$. The interface thickness is $2.5 \times 10^{-2}$. 

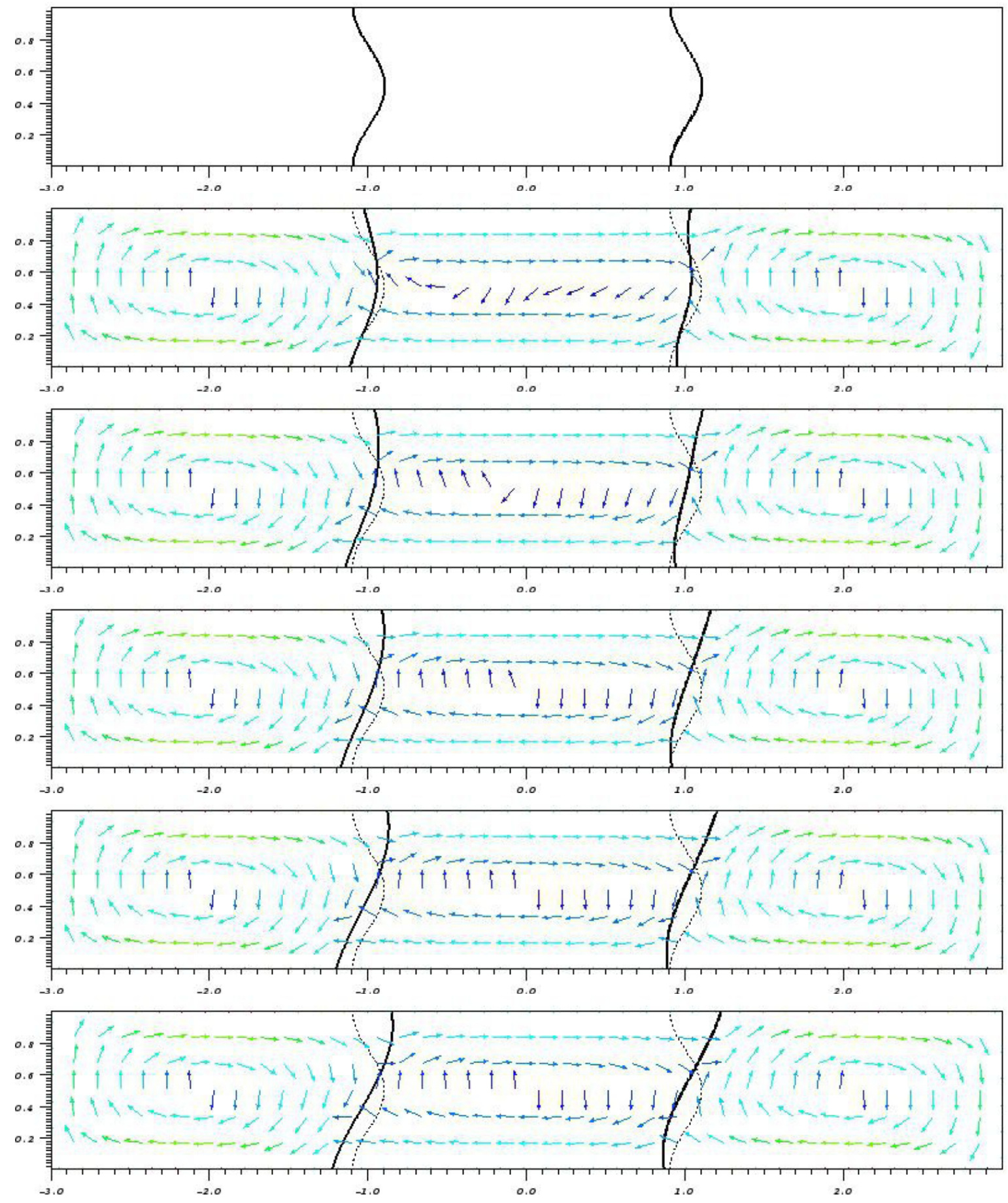

Figure 3. Asymmetric Couette flow with curved initial profile. The evolution of the interface is shown at times $0,0.5,1,1.5,2$ and 2.5. The arrows represent the velocity. The dotted line is the initial position of the interface. The material parameters are $\rho_{1} / \rho_{2}=10, \eta_{1}=\eta_{2}=10$, $\beta_{1}=1.5, \beta_{2}=0.6, M_{1}=M_{2}=0.01, \alpha=10^{-2}, \gamma=1$ and $\theta_{s}=68^{\circ}$. The shear velocity is $V=1.5$. The interface thickness is $2.5 \times 10^{-2}$. 

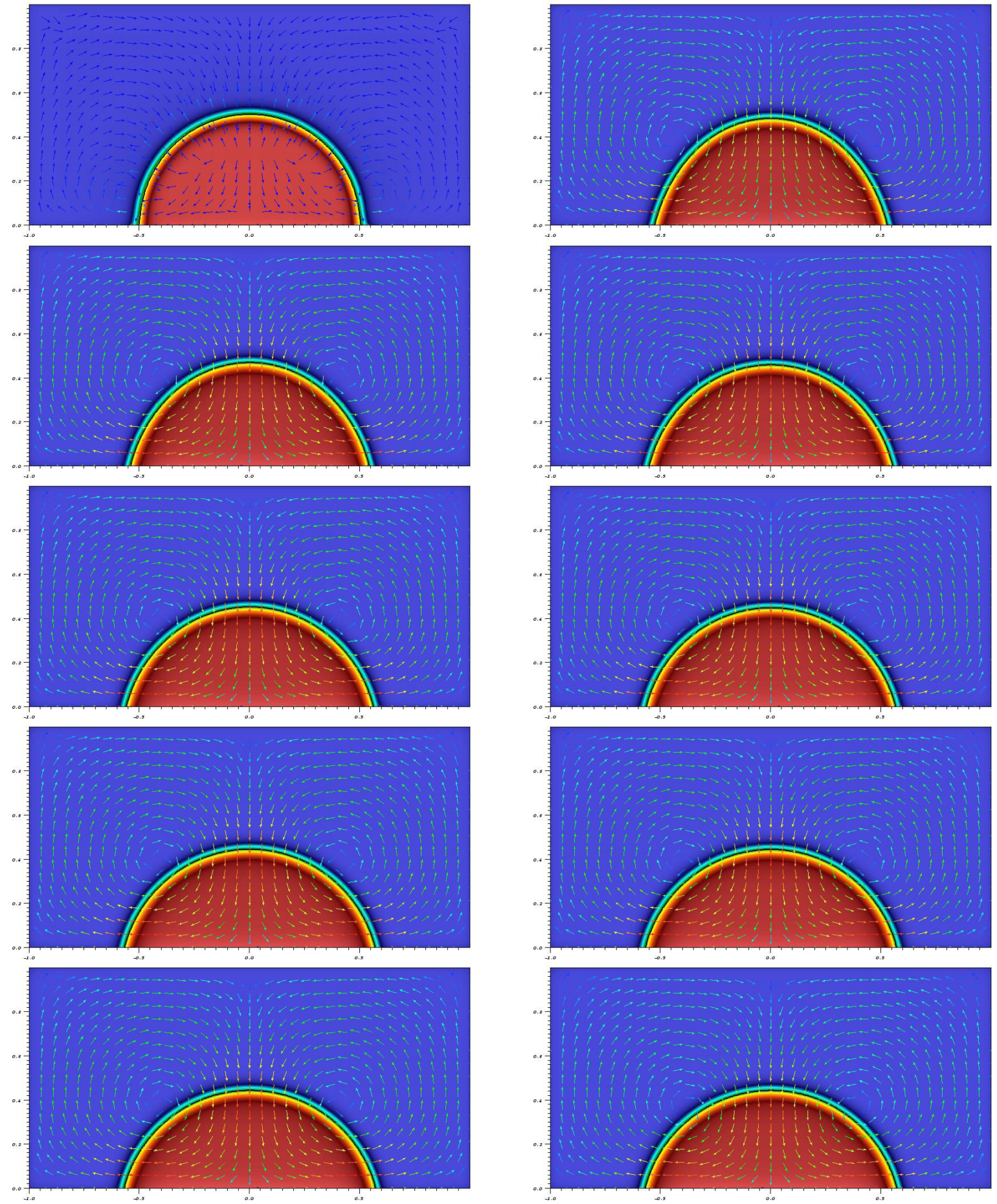

Figure 4. Evolution of a droplet with $\gamma=1, \theta_{s}=68^{\circ}$ without gravity. The interface is shown at times $0,0.5,1,1.5,2,2.5,3,3.5,4$, and 5 . Arrows represent the velocity field. $\rho_{2} / \rho_{1}=100$, $\eta_{2} / \eta_{1}=1 / 10, \beta_{1}=\beta_{2}=1.5, M_{1}=M_{2}=10^{-2}$, and $\alpha=10^{-2}$. The interface thickness is $\delta=2.5 \times 10^{-2}$. 

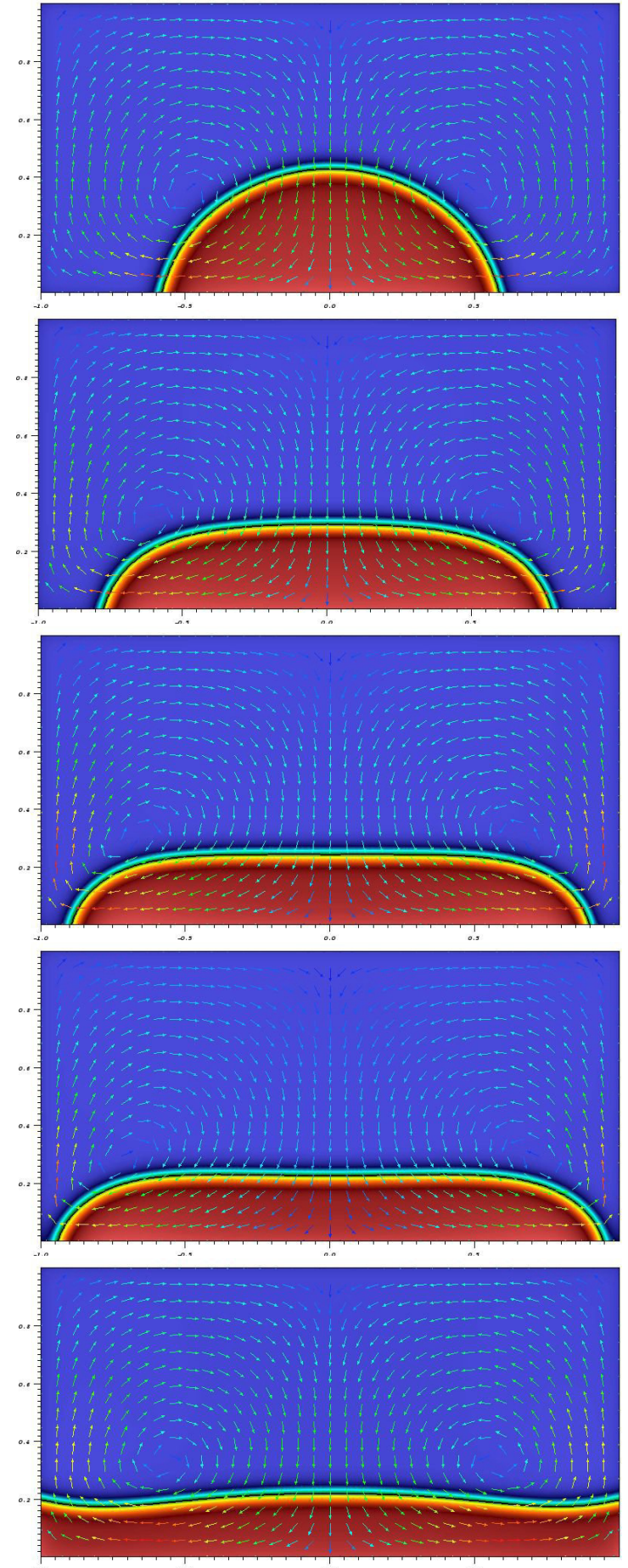

?.s.

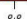
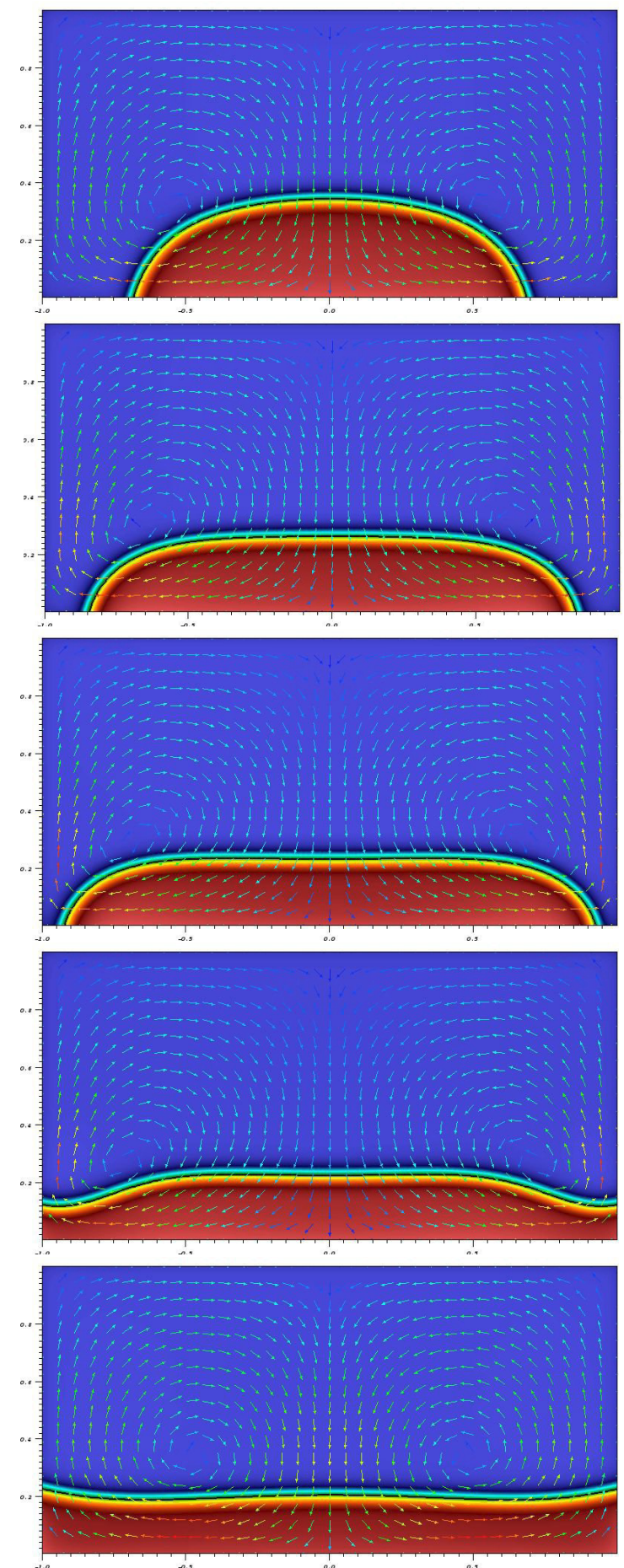

FiguRE 5. Evolution of a droplet with $\gamma=1, \theta_{s}=68^{\circ}$ with gravity. The interface is shown at times $0.5,1,1.5,2,2.5,3,3.5,4,4.5$ and 5 . Arrows represent the velocity field. $\rho_{2} / \rho_{1}=100$, $\eta_{2} / \eta_{1}=1 / 10, \beta_{1}=\beta_{2}=1.5, M_{1}=M_{2}=10^{-2}$, and $\alpha=10^{-2}$. The interface thickness is $\delta=2.5 \times 10^{-2}$. 

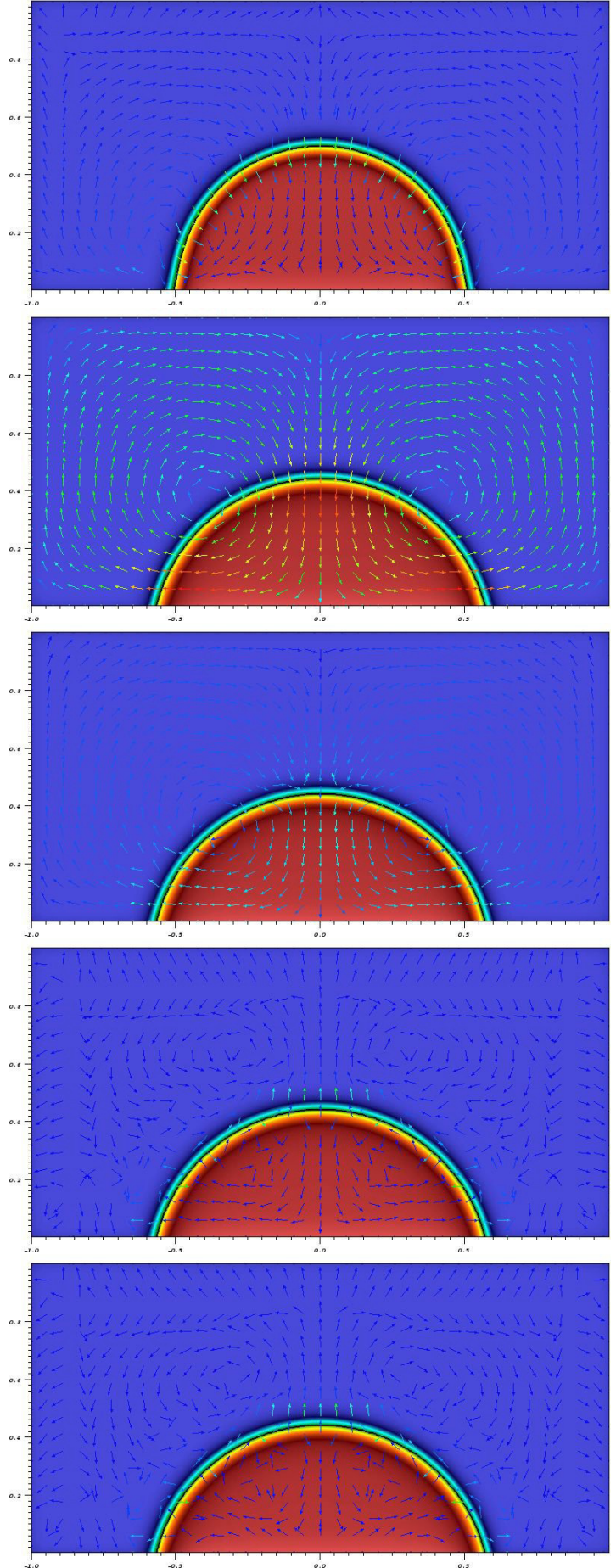
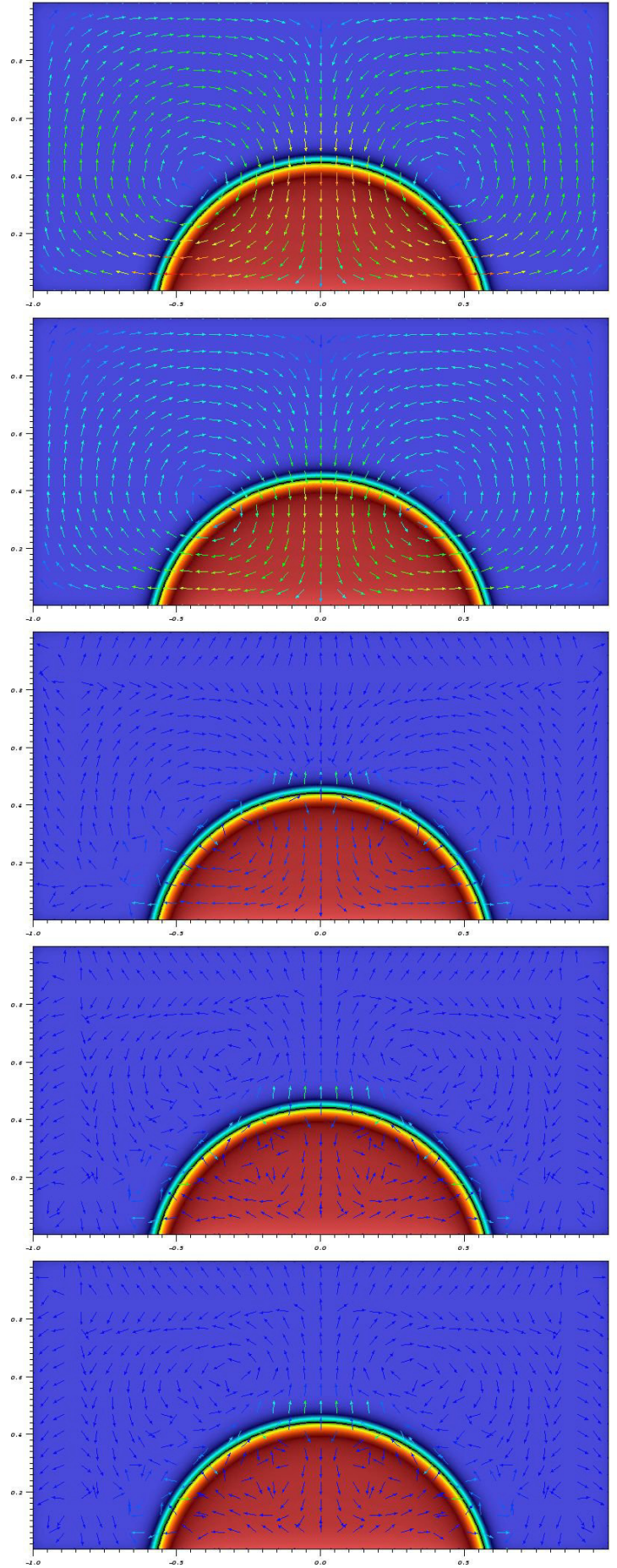

FigURE 6 . Evolution of a droplet with $\gamma=50, \theta_{s}=68^{\circ}$ without gravity. The interface is shown at times $0,0.5,1,1.5,2,2.5,3,3.5,4$, and 5 . Arrows represent the velocity field. $\rho_{2} / \rho_{1}=100$, $\eta_{2} / \eta_{1}=1 / 10, \beta_{1}=\beta_{2}=1.5, M_{1}=M_{2}=10^{-2}$, and $\alpha=10^{-2}$. The interface thickness is $\delta=2.5 \times 10^{-2}$. 

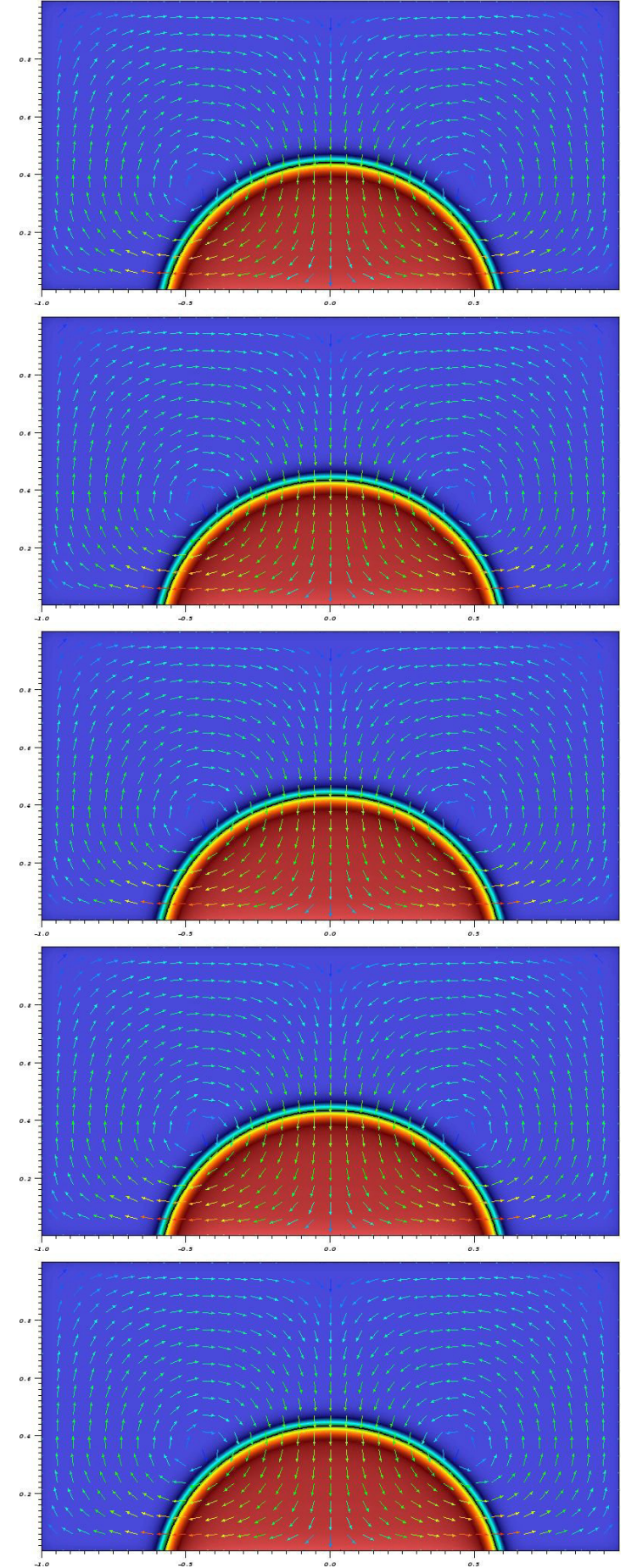

Figure 7. Evolution of a droplet with $\gamma=50, \theta_{s}=68^{\circ}$ with gravity. The interface is shown at times $0.5,1,1.5,2,2.5,3,3.5,4,4.5$ and 5 . Arrows represent the velocity field. $\rho_{2} / \rho_{1}=100$, $\eta_{2} / \eta_{1}=1 / 10, \beta_{1}=\beta_{2}=1.5, M_{1}=M_{2}=10^{-2}$, and $\alpha=10^{-2}$. The interface thickness is $\delta=2.5 \times 10^{-2}$.
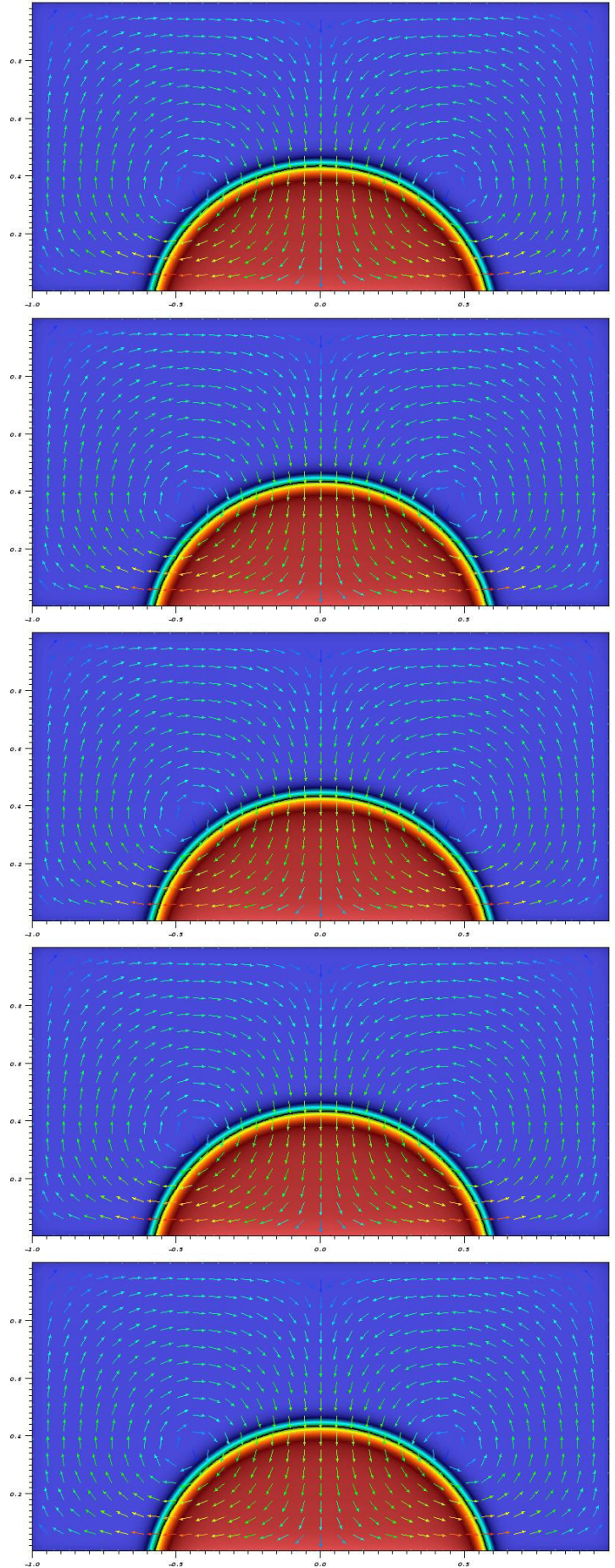

(1, 


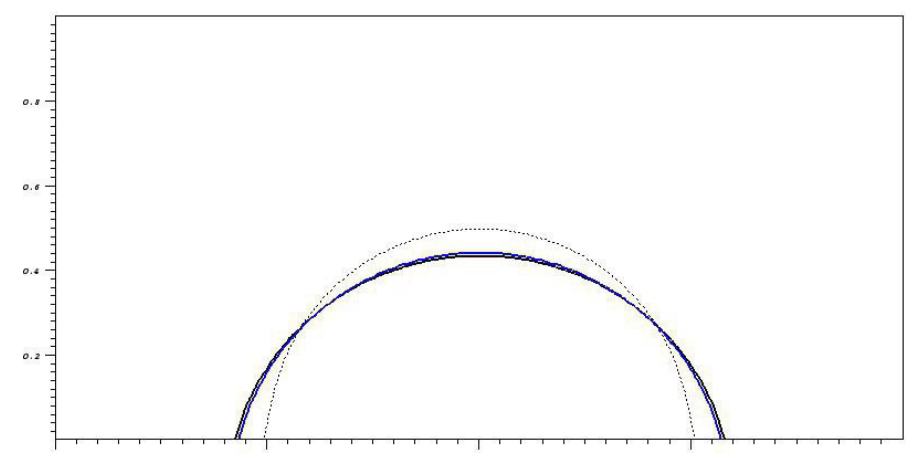

FiguRE 8. Comparison of the final state for a droplet for $\gamma=50$ and $\theta_{s}=68^{\circ}$ with and without gravity. $\rho_{2} / \rho_{1}=100, \eta_{2} / \eta_{1}=1 / 10, \beta_{1}=\beta_{2}=1.5, M_{1}=M_{2}=10^{-2}$, and $\alpha=10^{-2}$. The interface thickness is $\delta=2.5 \times 10^{-2}$. The black dotted line is the initial position of the interface. The black solid line is the final position of the interface under the action of gravity and the blue solid line the final position without gravity. Notice that, since the value of the surface tension is significantly large in comparison to gravity, the stationary shapes with and without gravity are the same and the contact angle changes in time.

\subsection{A Perturbed surface}

As in [26], we consider the evolution of a perturbed surface. The initial profile is given (in polar coordinates $(r, \varphi))$ by a perturbed semicircle $r=0.5+0.05 \sin (8 \varphi)$. The material parameters are as in Section 6.3 with $\gamma=10$ and $\theta_{s}=60^{\circ}$. The evolution is shown in Figure 9. As we can see, there is an initial period of time with fast dynamics, in which the surface tension rounds the interface. After which the droplet relaxes in a much similar way to Section 6.3.

\subsection{A Drop on an inclined plane}

Our last example studies the evolution of a droplet on an inclined plane under the action of gravity. The computational domain is

$$
\Omega=(0,1)^{2} \cup\left\{(x, y) \in \mathbb{R}^{2}: x \in(-1,0) y>|x|\right\} .
$$

The evolution at times $0,2,4,6,8,10,12,14,16,18,20,22,24,26,28,30,32$ and 34 is shown in Figure 10. The local mesh size away from the interface is about $10^{-2}$ and near it $8 \times 10^{-3}$. The material parameters are $\rho_{1} / \rho_{2}=50, \eta_{2} / \eta_{1}=1 / 10, \beta_{1}=\beta_{2}=1.5, M_{1}=M_{2}=10^{-2}$, and $\alpha=10^{-3} \cdot \gamma=10, \theta_{s}=90^{\circ}$. The time step is set to $\Delta t=5 \times 10^{-4}$ and the interface thickness equals $\delta=10^{-2}$. As we can see, the high value of the surface tension forces the droplet to adopt a certain shape before it is able to move a considerable distance. Since our model does not take into consideration contact angle hysteresis (see [9,41]), the droplet starts sliding instantaneously and keeps sliding. The same behavior can be observed for different inclination angles. For brevity, we do not show these results. Finally, it is worth noticing that the method is such that no special treatment for the interface is needed when it passes through the angle on the boundary which, clearly, indicates the robustness of the proposed scheme.

\subsection{Mass conservation}

One of the biggest concerns of practitioners regarding two-phase fluid simulation is mass conservation. Figure 11 is a plot of the area of the region $\left\{\phi_{h}^{k}>0\right\}$ against time, in the example of Section 6.5. This, if rescaled properly, can be interpreted as the mass of the droplet. As we can see, although the scheme does not 

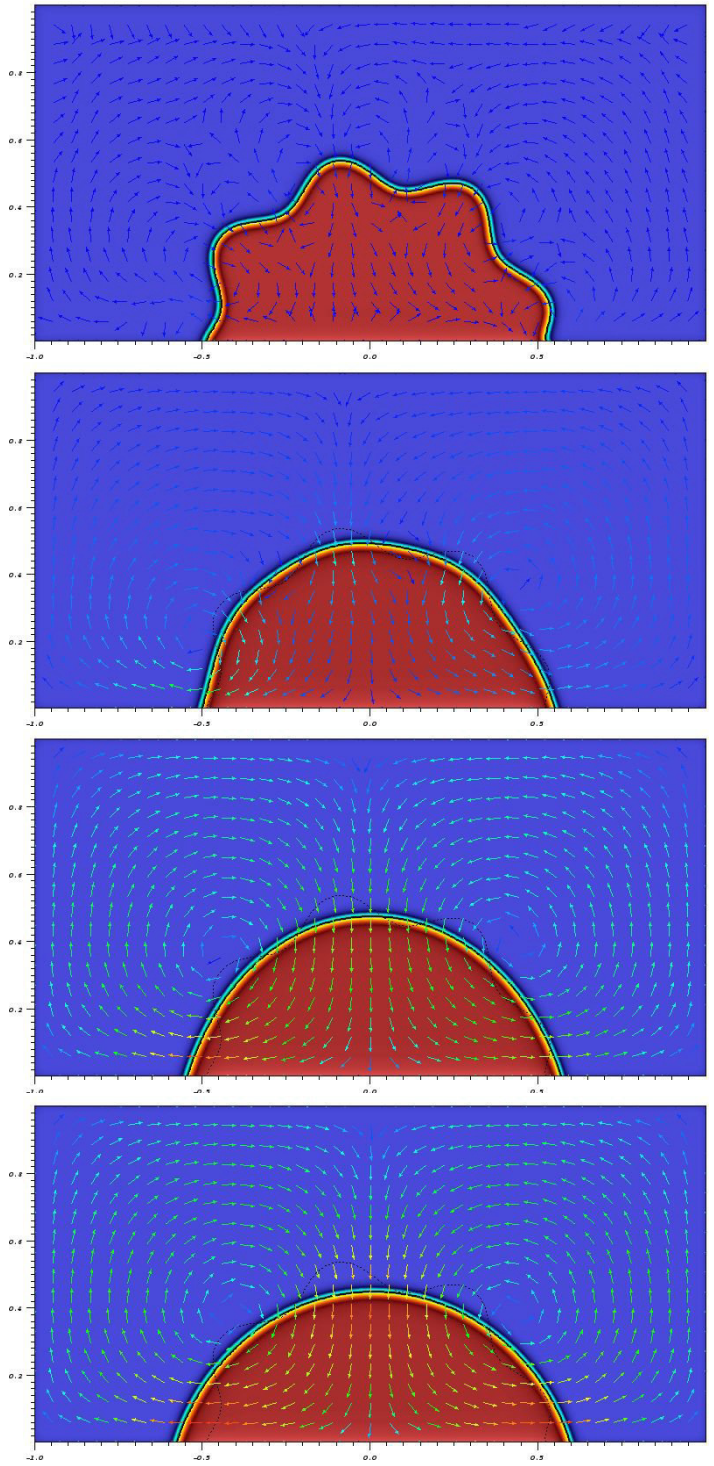
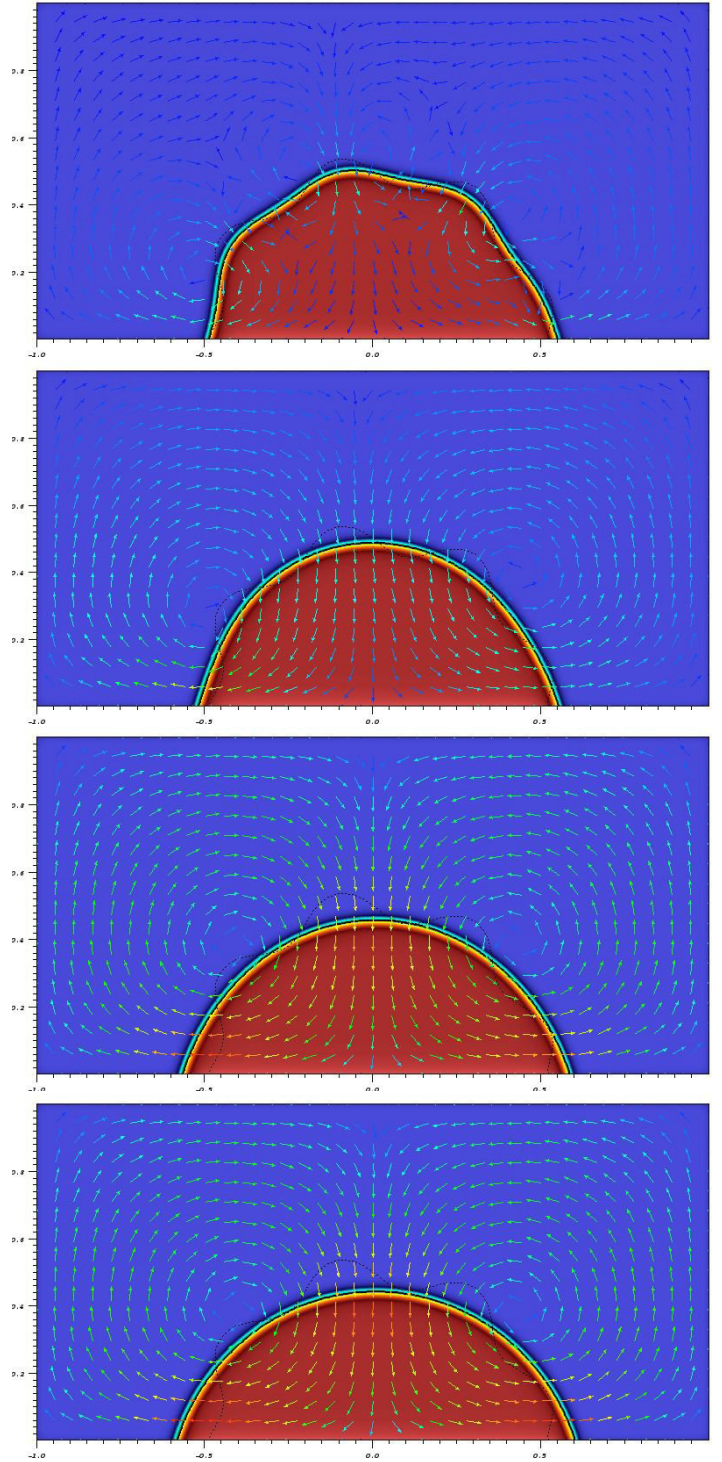

FIgURE 9. Evolution of an initially perturbed circular profile. The interface is shown at times $0,0.05,0.1,0.2,0.5,1,1.5,2$. Arrows represent the velocity field. $\rho_{2} / \rho_{1}=100, \eta_{2} / \eta_{1}=1 / 10$, $\beta_{1}=\beta_{2}=1.5, M_{1}=M_{2}=10^{-2}$, and $\alpha=10^{-2} \cdot \gamma=10, \theta_{s}=60^{\circ}$. The interface thickness is $\delta=2.5 \times 10^{-2}$.

preserve exactly the mass of the droplet, the relative variation between the initial and final areas is rather small, namely

$$
\frac{\left|\int_{\left\{\left.\phi\right|_{t=0}>0\right\}}-\int_{\left\{\left.\phi\right|_{t=T}>0\right\}}\right|}{\int_{\left\{\left.\phi\right|_{t=0}>0\right\}}} \times 100 \%=\frac{|0.128315-0.126055|}{0.128315} \times 100 \%=1.76 \% .
$$

It is interesting to notice the spike on the mass of the droplet at the time when it becomes in contact with the intersection between the inclined and horizontal surfaces. The whole derivation of the generalized Navier 

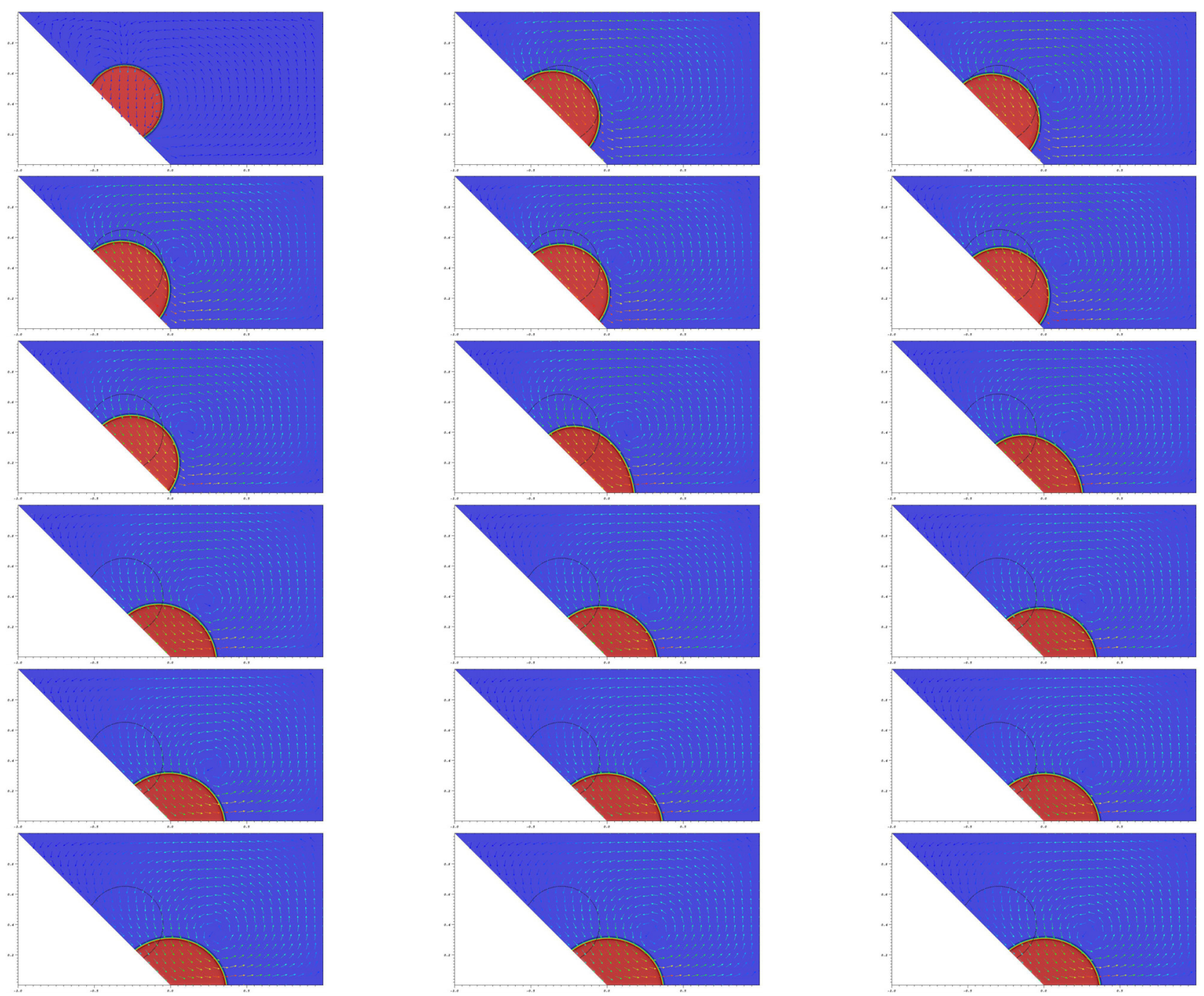

Figure 10. A droplet on an inclined plane. The evolution is shown at times $0,2,4,6,8,10$, $12,14,16,18,20,22,24,26,28,30,32$ and 34 . Arrows represent the velocity field and the black dotted line is the position of the interface at the beginning of the computations. The material parameters are $\rho_{1} / \rho_{2}=50, \eta_{2} / \eta_{1}=1 / 10, \beta_{1}=\beta_{2}=1.5, M_{1}=M_{2}=10^{-2}$, and $\alpha=10^{-3}$. $\gamma=10, \theta_{s}=90^{\circ}$ The interface thickness is $\delta=10^{-2}$.

boundary condition is based on the assumption that the surface is smooth enough so that it has a well defined normal, which is not the case at this point. The scheme, however, is able to handle this.

For comparison, Figure 12 shows the area of the region $\left\{\phi_{h}^{k}>0\right\}$ for the example in Section 6.4. In this case, the total change of mass is

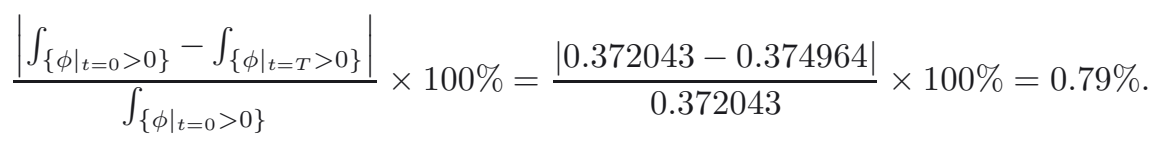




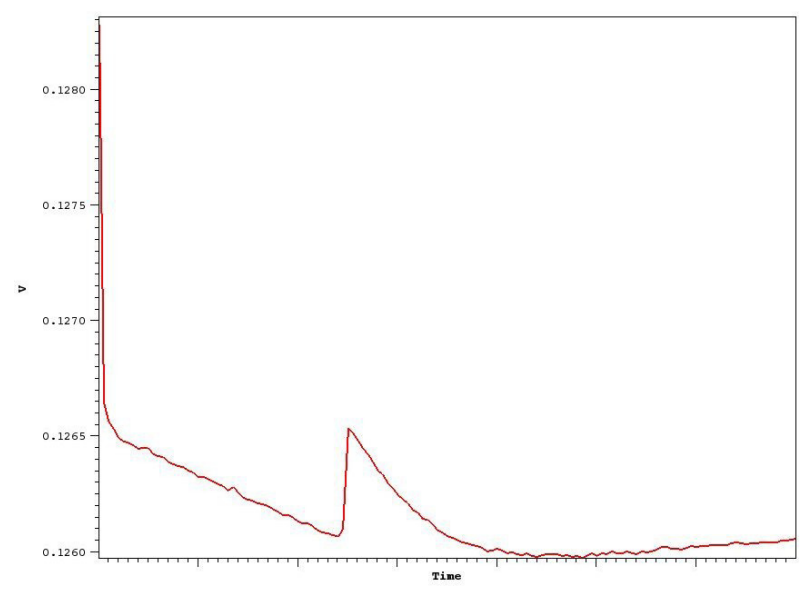

Figure 11. Total mass of the droplet vs. time for the problem of Section 6.5. The relative loss of mass is less than $1.76 \%$.

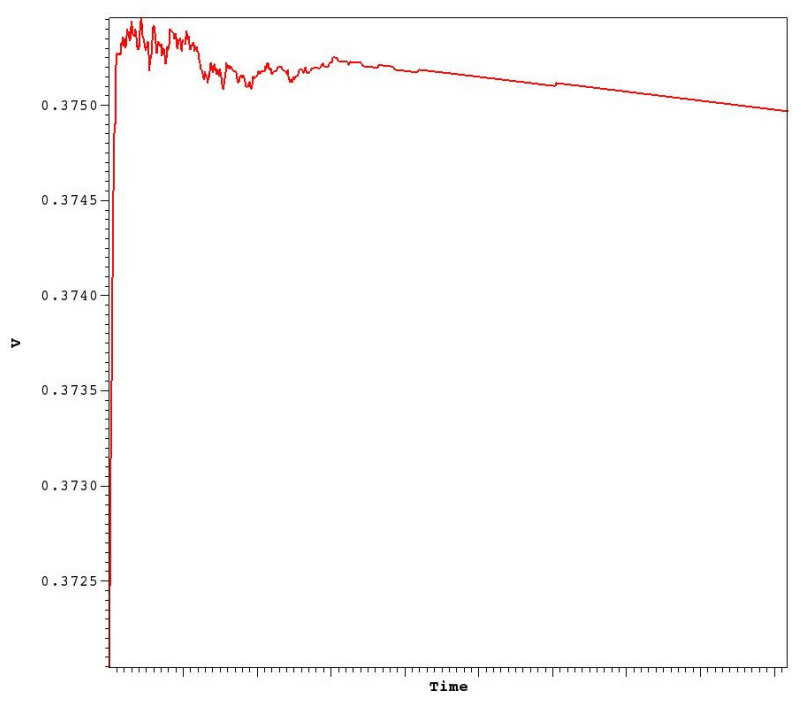

Figure 12. Total mass of the droplet vs. time for the problem of Section 6.4. The change in mass is less than $0.79 \%$.

\section{Conclusions And PERspectives}

In this work we have proposed a diffuse interface model for the movement of contact lines which consists of a Cahn Hilliard Navier Stokes system with nonstandard boundary conditions, and showed that this model has a natural energy law. We have proposed an efficient discretization technique based on operator splitting for Cahn Hilliard and fractional time-stepping based on penalization of the divergence for the Navier Stokes part. We showed that this scheme is unconditionally stable and presented a few numerical simulations to assess the capability of the developed approach.

We believe that the possible extensions of this approach are numerous. For instance, the addition of the so-called contact angle hysteresis (pinning) to the model is currently under investigation. This process is of crucial importance for practical applications. Moreover, we have developed a diffuse interface formulation for 
the problem of electrowetting on dielectric where this model is but a component of a much larger system of equations that describe this process. These results have been reported in [29].

Acknowledgements. The author would like to express his gratitude to Ricardo H. Nochetto (University of Maryland) for interesting discussions, carefully reading the original manuscript and making invaluable suggestions that helped improve the quality of the presentation.

\section{REFERENCES}

[1] H. Abels, H. Garcke and G. Grün, Thermodynamically consistent, frame indifferent diffuse interface models for incompressible two-phase flows with different densities. Math. Mod. Methods Appl. Sci. 22 (2012) 1150013.

[2] W. Bangerth, R. Hartmann and G. Kanschat, deal. II Differential Equations Analysis Library, Technical Reference. Available on http://www.dealii.org.

[3] W. Bangerth, R. Hartmann and G. Kanschat, deal.II - a general-purpose object-oriented finite element library. ACM Trans. Math. Softw. 33 (2007).

[4] T.D. Blake, The physics of moving wetting lines. J. Coll. Interf. Sci. 299 (2006) 1-13.

[5] T.D. Blake and Y.D. Shikhmurzaev, Dynamic wetting by liquids of different viscosity. J. Coll. Interf. Sci. 253 (2002) $196-202$.

[6] F. Boyer, C. Lapuerta, S. Minjeaud, B. Piar and M. Quintard, Cahn-Hilliard/Navier-Stokes model for the simulation of three-phase flows. Transp. Porous Media 82 (2010) 463-483.

[7] F. Brezzi and M. Fortin, Mixed and Hybrid Finite Element Methods. Springer-Verlag, New York, NY (1991).

[8] L.A. Caffarelli and N.E. Muler, An $L^{\infty}$ bound for solutions of the Cahn-Hilliard equation. Arch. Ration. Mech. Anal. 133 (1995) 129-144.

[9] Antonio DeSimone, Natalie Grunewald and Felix Otto, A new model for contact angle hysteresis. Netw. Heterog. Media 2 (2007) 211-225.

[10] J.-B. Dupont and D. Legendre, Numerical simulation of static and sliding drop with contact angle hysteresis. J. Comput. Phys. 229 (2010) 2453-2478.

[11] J. Eggers and R. Evans, Comment on "dynamic wetting by liquids of different viscosity," by t.d. blake and y.d. shikhmurzaev. J. Coll. Interf. Sci. 280 (2004) 537-538.

[12] A. Ern and J.-L. Guermond, Theory and practice of finite elements, Applied Mathematical Sciences. Springer-Verlag, New York $159,2004$.

[13] Xiaobing Feng, Fully discrete finite element approximations of the Navier-Stokes-Cahn-Hilliard diffuse interface model for two-phase fluid flows. SIAM J. Numer. Anal. 44 (2006) 1049-1072.

[14] M. Gao and X.-P. Wang, A gradient stable scheme for a phase field model for the moving contact line problem. J. Comput. Phys. 231 (2012) 1372-1386.

[15] J.-F. Gerbeau and T. Lelièvre, Generalized Navier boundary condition and geometric conservation law for surface tension. Comput. Methods Appl. Mech. Engrg. 198 (2009) 644-656.

[16] V. Girault and P.-A. Raviart, Finite Element Methods for Navier-Stokes Equations. Theory and Algorithms. Springer Series in Computational Mathematics. Springer-Verlag, Berlin, Germany (1986).

[17] J.-L. Guermond, P. Minev and J. Shen, Error analysis of pressure-correction schemes for the Navier-Stokes equations with open boundary conditions. SIAM J. Num. Anal. 43 (2005) 239-258.

[18] J.-L. Guermond and L. Quartapelle, A projection FEM for variable density incompressible flows. J. Comput. Phys. 165 (2000) $167-188$.

[19] J.-L. Guermond and A. Salgado, A splitting method for incompressible flows with variable density based on a pressure Poisson equation. J. Comput. Phys. 228 (2009) 2834-2846.

[20] Q. He, R. Glowinski and X.-P. Wang, A least-squares/finite element method for the numerical solution of the Navier-StokesCahn-Hilliard system modeling the motion of the contact line. J. Comput. Phys. 230 (2011) 4991-5009.

[21] C. Huh and L.E. Scriven, Hydrodynamic model of steady movement of a solid/liquid/fluid contact line. J. Coll. Interf. Sci. 35 (1971) 85-101.

[22] D. Jacqmin, Calculation of two-phase Navier-Stokes flows using phase-field modeling. J. Comput. Phys. 155 (1999) 96-127.

[23] D. Kay, V. Styles and R. Welford, Finite element approximation of a Cahn-Hilliard-Navier-Stokes system. Interfaces Free Bound. 10 (2008) 15-43.

[24] D. Kay and R. Welford, Efficient numerical solution of Cahn-Hilliard-Navier-Stokes fluids in 2D. SIAM J. Sci. Comput. 29 (2007) 2241-2257.

[25] J. Kim, K. Kang and J. Lowengrub, Conservative multigrid methods for Cahn-Hilliard fluids. J. Comput. Phys. 193 (2004) $511-543$.

[26] Z. Li, M.-C. Lai, G. He and H. Zhao, An augmented method for free boundary problems with moving contact lines. Comput. 85 Fluids 39 (2010) 1033-1040.

[27] S. Manservisi and R. Scardovelli, A variational approach to the contact angle dynamics of spreading droplets. Comput. ES Fluids 38 (2009) 406-424. 
[28] S. Minjeaud, An unconditionally stable uncoupled scheme for a triphasic Cahn-Hilliard/Navier-Stokes model. Numer. Methods Partial Differ. Eqn. (2012).

[29] R.H. Nochetto, A.J. Salgado and S.W. Walker, A diffuse interface model for electrowetting on dielectric with moving contact lines (2011). Submitted to M3AS.

[30] C.E. Norman and M.J. Miksis, Gas bubble with a moving contact line rising in an inclined channel at finite Reynolds number. Phys. D 209 (2005) 191-204.

[31] T. Qian, X.-P. Wang and P. Sheng, Generalized Navier boundary condition for the moving contact line. Commun. Math. Sci. 1 (2003) 333-341.

[32] T. Qian, X.-P. Wang and P. Sheng, Molecular hydrodynamics of the moving contact line in two-phase immiscible flows. Commun. Comput. Phys. 1 (2006) 1-52.

[33] T. Qian, X.-P. Wang and P. Sheng, A variational approach to moving contact line hydrodynamics. J. Fluid Mech. 564 (2006) $333-360$.

[34] W. Ren and W.E, Boundary conditions for the moving contact line problem. Phys. Fluids 19 (2007) 022101.

[35] J. Shen and X. Yang, Energy stable schemes for Cahn-Hilliard phase-field model of two-phase incompressible flows. Chin. Ann. Math. Ser. B 31 (2010) 743-758.

[36] J. Shen and X. Yang, Numerical approximations of Allen-Cahn and Cahn-Hilliard equations. Discrete Contin. Dyn. Syst. 28 (2010) 1669-1691.

[37] J. Shen and X. Yang, A phase-field model and its numerical approximation for two-phase incompressible flows with different densities and viscosities. SIAM J. Sci. Comput 32 (2010) 1159-1179.

[38] Y.D. Shikhmurzaev, Capillary flows with forming interfaces. Chapman \& Hall/CRC, Boca Raton, FL (2008).

[39] Y.D. Shikhmurzaev and T.D. Blake, Response to the comment on [J. Colloid Interface Sci. 253 (2002) 196] by J. Eggers and R. Evans, J. Coll. Interf. Sci. 280 (2004) 539-541.

[40] P.D.M. Spelt, A level-set approach for simulations of flows with multiple moving contact lines with hysteresis. J. Comput. Phys. 207 (2005) 389-404.

[41] A. Turco, F. Alouges and A. DeSimone, Wetting on rough surfaces and contact angle hysteresis: numerical experiments based on a phase field model. ESAIM: M2AN 43 (2009) 1027-1044.

[42] S.M. Wise, C. Wang and J.S. Lowengrub, An energy-stable and convergent finite-difference scheme for the phase field crystal equation. SIAM J. Numer. Anal. 47 (2009) 2269-2288.

[43] E. Zeidler, Nonlinear functional analysis and its applications. I. Fixed-point theorems, Translated from the German by Peter R. Wadsack. Springer-Verlag, New York (1986). 